\title{
One-loop Helicity Amplitudes for Top Quark Pair Production in Randall-Sundrum Model
}

\author{
Hua Xing Zhu, Chong Sheng Li:* Liang Dai, Jun Gao, and Jian Wang \\ Department of Physics and State Key Laboratory of Nuclear Physics and Technology, \\ Peking University, Beijing 100871, China \\ C.-P.Yuant \\ Department of Physics and Astronomy, \\ Michigan State University, East Lansing, MI 48824, USA
}

\begin{abstract}
In this paper, we show how to calculate analytically the one-loop helicity amplitudes for the process $q \bar{q} \rightarrow t \bar{t}$ induced by KK gluon, using the spinor helicity formalism. A minimal set of Feynman rules which are uniquely fixed by gauge invariance and the color representation of the KK gluon are derived and used in the calculation. Our results can be applied to a variety of models containing a massive color octet vector boson.
\end{abstract}

*Electronic address: csli@pku.edu.cn

$\dagger$ Electronic address: yuan@pa.msu.edu 


\section{INTRODUCTION}

Warped extra-dimension model proposed by Randall and Sundrum (RS) [1] is a popular model that can solve the hierarchy problem. When allowing the Standard Model (SM) field propagating in the extra dimension, the RS model provides many novel points of view for some problems, like hierarchy of fermion masses and the unification of gauge couplings.

The first signal of the RS model may be the observation of KK gluon (first Kaluza-Klein excitation mode of SM gluon) as a resonant in the $t \bar{t}$ final state at the early LHC, simply because its production rate is large compare to the other KK particles [2]. Detailed study of total cross sections and the invariant mass distribution of $t \bar{t}$ production induced by KK gluon have been made in the literatures [2 -8] at the Leading Order (LO) in QCD.

Recently CDF collaboration reported a large top quark forward-backward asymmetry with integrated luminosity $5.3 \mathrm{fb}^{-1}[9]$. This $3.4 \sigma$ discrepancy with SM predictions [10, 11] has motivated a lot of work in the theory community, cf. ref. [12] and references therein.

One attractive explanation of the large anomalous forward-backward asymmetry is provided by the RS model. Within the RS framework, a realistic model has been constructed that has been shown to be able to explain the forward-backward asymmetry of top quark sector as well as bottom quark sector simultaneously [13]. This is realized by a careful choice of fermion localizations, so that there is a relatively light KK gluon with mass around 1.5 $\mathrm{TeV}$ and large parity violation in the first generation of quark couplings. The authors of Ref. [13] have shown at the LO in QCD that the model they constructed can explain well the total asymmetry observed at the Tevatron by CDF [9], as well as the asymmetry at large top quark pair invariant mass region and large rapidity region [9].

It's well known that LO prediction suffers from large scale uncertainty, and therefore is not appropriate for precision measurement, e.g., extraction of couplings between quark and KK gluon. Furthermore, $t \bar{t}$ production is known to have large K-factor at the Next-toLeading Order (NLO) in QCD [14-16]. The large K-factor is partially taken into account in the Ref. [13] using a formalism proposed in [17]. Aiming at the precise prediction for the KK gluon mediated $t \bar{t}$ production, Ref. [18] calculated the interference of SM box diagrams and tree diagram of $t \bar{t}$ production induced by KK gluon. Furthermore, Ref. [19] consider the effects of KK gluon on $t \bar{t}$ production by gluon-gluon fusion. It was found that the contributions, although non-zero, are highly suppressed. For this reason, we focus our 
attention the $q \bar{q}$ channel in the current work.

In this paper, we investigate how to calculate the complete NLO QCD corrections to $t \bar{t}$ production induced by KK gluon, which has not been reported in the previous literature. We first isolate a minimal set of gauge invariant interactions and derive the relevant Feynman rules for the KK gluon, the ghost of KK gluon and the 5th component of the five-dimension (5D) KK gluon field in $R_{\xi}$ gauge, and show in detail how to renormalize the resulting one-loop amplitudes. Finally we present the full one-loop helicity amplitudes for KK gluon induced $t \bar{t}$ production, and the contribution from real emissions at the NLO is left to be done in another work. As a by-product, we also calculate the NLO total decay width of KK gluon in the large $m_{K K}$ limit. A complete numerical result and phenomenology discussion of the NLO corrections to $t \bar{t}$ production induced by KK gluon will be presented elsewhere [20].

This paper is organized as follows: in Sec. II we briefly derive the relevant Feynman rules used in our calculation. Details of the one-loop calculation are presented in sec. III, A discussion on the result and a brief conclusion is given in sec. IV] All relevant Feynman rules can be found in appendix $\mathrm{A}$,

\section{THE MODEL}

In this section we derive the Feynman rules relevant in our calculation. Part of the results in this section are well known in the literatures [21 28]. The RS construction is a slice of 5 dimensional Anti-de Sitter space. Since gravitational fluctuations play no role in the problem, we consider a fixed background metric of the form

$$
d s^{2}=\frac{1}{k^{2} z^{2}}\left(\eta_{\mu \nu} d x^{\mu} d x^{\nu}-d z^{2}\right)
$$

where $z$ lives on the interval $\left[z_{1}=1 / k, z_{2}=1 / T\right]$. It's assumed that $k$ is of the order $M_{P l}$, and $T$ is of the order $\mathrm{TeV}$.

The action for a 5 dimensional massless gauge boson with $S U(3)$ gauge symmetry is

$$
S_{5 D}=\int d^{4} x d z \sqrt{G}\left(-\frac{1}{2} \operatorname{Tr} F_{M N} F^{M N}\right),
$$

where the Roman indices $M, N$ run from $0 \ldots 3,5$. The field strength for the $5 \mathrm{D}$ gauge field is defined as

$$
F_{M N}^{a}=\partial_{M} A_{N}^{a}-\partial_{N} A_{M}^{a}+g_{5} f^{a b c} A_{M}^{b} A_{N}^{c}, \quad F_{M N}=F_{M N}^{a} T^{a},
$$


where $g_{5}$ is the gauge coupling constant in $5 \mathrm{D} . T^{a}$ is the conventional Gell-mann matrix with normalization $\operatorname{Tr}\left[T^{a} T^{b}\right]=\frac{1}{2} \delta^{a b}$. Writing the action in terms of $4 \mathrm{D}$ components and the 5 th component of the gauge field, we have

$$
S_{5 D}=\int d^{4} x \int \frac{d z}{k z}\left[-\frac{1}{4} F_{\mu \nu}^{a} F^{\mu \nu, a}-\frac{1}{2} F_{\mu 5}^{a} F^{\mu 5, a}\right] .
$$

It can be seen from Eq. (44) that there is mixing between the $4 \mathrm{D}$ components $A_{\mu}$ and $A_{5}$. To cancel the quadratic mixing terms, it's conventional to introduce the following bulk and boundary gauge fixing terms

$$
\begin{gathered}
S_{G F, b u l k}=\int d^{4} x \int \frac{d z}{k z}\left(-\frac{1}{2 \xi}\right)\left[\partial^{\mu} A_{\mu}^{a}-\xi(k z) \partial_{z}\left(\frac{1}{k z} A_{5}^{a}\right)\right]^{2}, \\
S_{G F, \text { boundary }}=-\frac{1}{2 \xi_{b}} \int d^{4} x\left[\left.\left(\partial^{\mu} A_{\mu}^{a}+\xi_{b} \frac{1}{k z} A_{5}^{a}\right)^{2}\right|_{z=z_{2}}+\left.\left(\partial^{\mu} A_{\mu}^{a}-\xi_{b} \frac{1}{k z} A_{5}^{a}\right)^{2}\right|_{z=z_{1}}\right] .
\end{gathered}
$$

A convenient gauge choice for the boundary terms is the unitary gauge $\xi_{b} \rightarrow \infty$, in which the boundary condition for the gauge field is

$$
\left.\partial_{z} A^{\mu, a}\right|_{z=z_{1}, z_{2}}=0,\left.\quad A_{5}^{a}\right|_{z=z_{1}, z_{2}}=0 .
$$

As usual in quantizing spin-1 gauge fields, we also need to introduce ghost field to appropriately account for the degrees of freedom. Following the Faddeev-Popov procedure, the 5D ghost Lagrangian in $R_{\xi}$ gauge is

$$
S_{5 D, \text { ghost }}=\int d^{4} x \int \frac{d z}{k z} \bar{u}^{a}\left[-\partial^{\mu} \mathcal{D}_{\mu}+\xi(k z) \partial_{z} \frac{1}{k z} \partial_{z}\right]^{a b} u^{b},
$$

where $\mathcal{D}_{\mu}$ is the covariant derivative in adjoint representation:

$$
\left(\mathcal{D}_{M}\right)^{a b}=\delta^{a b} \partial_{M}+g_{5} f^{a c b} A_{M}^{c}
$$

The final action is then given by

$$
S=S_{5 D}+S_{G F, b u l k}+S_{G F, b o u n d a r y}+S_{5 D, \text { ghost }}
$$

To derive the relevant Feynman rules, we expand the gauge field in terms of a set of orthonormal KK modes:

$$
\begin{aligned}
& A_{\mu}(x, z)=\sqrt{k} \sum_{j=0}^{\infty} A_{\mu}^{(j)}(x) \chi_{j}(z) \\
& A_{5}(x, z)=\sqrt{k} \sum_{j=1}^{\infty} A_{5}^{(j)}(x) \frac{1}{m_{j}} \partial_{z} \chi_{j}(z),
\end{aligned}
$$


where the orthonomal basis $\chi_{j}$ satisfies

$$
\int \frac{d z}{z} \chi_{i}(z) \chi_{j}(z)=\delta_{i j}
$$

and is determined by

$$
\begin{aligned}
& \partial_{z}\left(\frac{1}{z} \partial_{z} \chi_{j}(z)\right)+\frac{m_{j}^{2}}{z} \chi_{j}(z)=0 \\
& \left.\partial_{z} \chi_{j}(z)\right|_{z=z_{1}, z_{2}}=0
\end{aligned}
$$

The ghost field has similar KK decomposition in terms of 4D ghost field:

$$
\begin{aligned}
& u(x, z)=\sqrt{k} \sum_{j=0}^{\infty} u^{(j)}(x) \chi_{j}(z), \\
& \bar{u}(x, z)=\sqrt{k} \sum_{j=0}^{\infty} \bar{u}^{(j)}(x) \chi_{j}(z) .
\end{aligned}
$$

Substituting the expansion, Eq. (11), into the action, Eq. (4), and integrating over the 5th dimension, we obtain the 4D Lagrangian that describes the interaction of various 4D field. The first few KK modes that are relevant to our discussion are $A_{\mu}^{(0)}, A_{\mu}^{(1)}, A_{5}^{(1)}, u^{(0)}$ and $u^{(1)}$. The propagator of these fields are given as, in $R_{\xi}$ gauge:

$$
\begin{aligned}
& a, \mu \underset{p}{\operatorname{eecele}} b, \nu=-\frac{i \delta^{a b}}{p^{2}}\left(g^{\mu \nu}-\frac{p^{\mu} p^{\nu}}{p^{2}}(1-\xi)\right), \quad A_{\mu}^{(0)}
\end{aligned}
$$

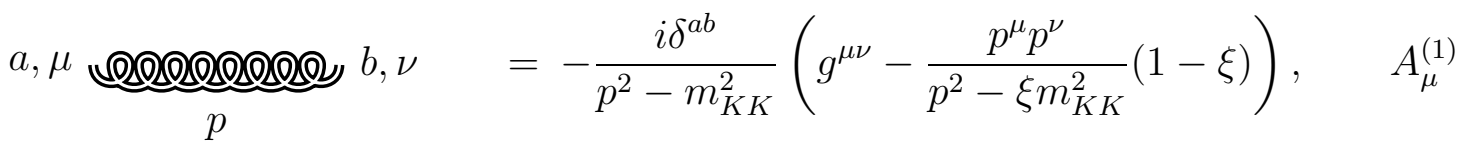

$$
\begin{aligned}
& \begin{array}{c}
a-\cdots--b \\
p
\end{array} \quad=\frac{i \delta^{a b}}{p^{2}-\xi m_{K K}^{2}}, \quad A_{5}^{(1)} \\
& a \cdots \cdots b \cdot \quad=\frac{i \delta^{a b}}{p^{2}}, \quad u^{(0)} \\
& a \bullet \cdots \cdots \cdots b \quad=\frac{i \delta^{a b}}{p^{2}-\xi m_{K K}^{2}}, \quad u^{(1)}
\end{aligned}
$$

where we have identified the zero KK mode as the QCD gluon field, and denote the mass of first $\mathrm{KK}$ mode as $m_{K K}$. Without confusion, we also call the first KK mode as KK gluon occasionally. It's a straightforward exercise to derive the Feynman vertices for these fields. For example, the vertex of 3 zero KK mode comes from the Lagrangian

$$
\mathcal{L}_{3 g}=\int \frac{\mathrm{d} z}{k z}\left(-\frac{1}{2} g_{5} f^{a b c} A_{\mu}^{(0), b} A_{\nu}^{(0), c}\left(\partial_{\mu} A_{\nu}^{(0), a}-\partial_{\nu} A_{\mu}^{(0), a}\right)\right)(\sqrt{k})^{3} \chi_{0}^{3}(z) .
$$


The $z$ integral can be trivially done since $\chi_{0} \equiv \chi_{0}(z)$ is a constant, and

$$
\int \frac{\mathrm{d} z}{k z}(\sqrt{k})^{3} \chi_{0}^{3}(z)=\sqrt{k} \chi_{0}
$$

It's immediately clear that one can identify the QCD gauge coupling as $g_{s}=\sqrt{k} \chi_{0} g_{5}$, so that $\mathcal{L}_{3 g}$ gives the conventional 3-point gluon interaction in QCD. One interesting feature of $\mathcal{L}_{3 g}$ is that the resulting coupling is independent of profile of the KK mode in the 5 th dimension. Actually one can derive a set of vertices that have this feature from the action in Eq. (10). Part of these vertices are just the conventional QCD vertices. The other part describes the interaction between the zero KK mode and the first KK mode, which can be found in the appendix.

Finally, we need to know the interaction between the KK mode and fermion, which is sensitive to the 5 th dimension profile of the KK mode. This can be done by adding a term describing 5D fermion interaction with 5D gauge boson field into Eq. (10):

$$
\begin{aligned}
S_{5 D, \text { fermion }} & =\left.\int d^{5} x \sqrt{-g}\left\{i \bar{\Psi} \Gamma^{M} D_{M} \Psi\right\}\right|_{\bar{\Psi} A \Psi \text { piece }} \\
& =\int d^{4} x \int d z\left(\frac{1}{k z}\right)^{4} \bar{\Psi}\left[g_{5} \gamma^{\mu} A_{\mu}+i g_{5} \gamma^{5} A_{5}\right] \Psi \\
& =\int d^{4} x \int d z\left(\frac{1}{k z}\right)^{4} g_{5}\left\{\psi \sigma^{\mu} A_{\mu} \bar{\psi}+\bar{\chi} \bar{\sigma}^{\mu} A_{\mu} \chi+i(-\psi \chi+\bar{\chi} \bar{\psi}) A_{5}\right\}
\end{aligned}
$$

where the covariant derivative is defined as

$$
\begin{aligned}
D_{\mu} \Psi & =\left(\partial_{\mu}-\frac{i}{2 z} \gamma_{\mu} \gamma_{5}\right) \Psi, \\
D_{5} \Psi & =\partial_{z} \Psi
\end{aligned}
$$

and

$$
\Psi=\left(\begin{array}{c}
\chi \\
\bar{\psi}
\end{array}\right)
$$

is a Dirac spinor. The 5D fermion field can be expanded in terms of fermion KK modes:

$$
\begin{aligned}
& \chi(x, z)=\sum_{j=0} g_{j}(z) \chi^{(j)}(x), \\
& \bar{\psi}(x, z)=\sum_{j=1} f_{j}(z) \bar{\psi}^{(j)}(x),
\end{aligned}
$$

where $g_{j}(z)$ and $f_{j}(z)$ are the fermion wave functions of the 5 th dimension, with the normalization

$$
\int d z\left(\frac{1}{k z}\right)^{4} g_{n}^{2}(z)=\int d z\left(\frac{1}{k z}\right)^{4} f_{n}^{2}(z)=1
$$


Substituting the expansion into Eq. (18), we derive the interaction between fermion zero mode and KK zero mode:

$$
\int d^{4} x g_{s}\left\{\psi^{(0)} \sigma^{\mu} A_{\mu}^{(0)} \bar{\psi}^{(0)}+\bar{\chi}^{(0)} \bar{\sigma}^{\mu} A_{\mu}^{(0)} \chi^{(0)}\right\}
$$

This is just the conventional QCD interaction between fermion and gluon. For the interaction between the fermion zero mode and first KK mode, we have

$$
\int d^{4} x\left\{C_{R} \psi^{(0)} \sigma^{\mu} A_{\mu}^{(1)} \bar{\psi}^{(0)}+C_{L} \bar{\chi}^{(0)} \bar{\sigma}^{\mu} A_{\mu}^{(1)} \chi^{(0)}\right\}
$$

where the chiral couplings are defined as

$$
C_{L}=\sqrt{k} g_{5} \int d z\left(\frac{1}{k z}\right)^{4} g_{0}^{2}(z) \chi_{1}(z), \quad C_{R}=\sqrt{k} g_{5} \int d z\left(\frac{1}{k z}\right)^{4} f_{0}^{2}(z) \chi_{1}(z) .
$$

There are also interaction terms between fermion zero mode and $A_{5}^{(1)}$ :

$$
\begin{aligned}
& \int d^{4} x \int d z\left(\frac{1}{k z}\right)^{4} i g_{5} g_{0}(z) f_{0}(z)\left(-\psi^{(0)} \chi^{(0)}+\bar{\chi}^{(0)} \bar{\psi}^{(0)}\right) \sqrt{k} A_{5}^{(1)} \frac{1}{m_{K K}} \partial_{z} \chi_{1}(z) \\
= & \int d^{4} x i g_{5} \frac{\sqrt{k}}{m_{K K}}\left(-\psi^{(0)} \chi^{(0)}+\bar{\chi}^{(0)} \bar{\psi}^{(0)}\right) A_{5}^{(1)} \int d z\left(\frac{1}{k z}\right)^{4} g_{0}(z) f_{0}(z) \partial_{z} \chi_{1}(z),
\end{aligned}
$$

where all the $z$ dependencies have been written out explicitly. Also the 5 th dimension wave function of gauge field $\chi_{1}(z)$ should not be confused with the chiral fermion field $\chi^{(0)}(x)$. Integrating by part over the $z$ integral, we obtain

$$
\begin{aligned}
& \int d z\left(\frac{1}{k z}\right)^{4} g_{0}(z) f_{0}(z) \partial_{z} \chi_{1}(z) \\
= & {\left[\left(\frac{1}{k z}\right)^{4} g_{0} f_{0} \partial_{z} \chi_{1}\right]_{z=z_{1}}^{z=z_{2}}-\int d z\left(\frac{1}{k z}\right)^{4}\left[-\frac{4}{z} g_{0} f_{0}+g_{0}^{\prime} f_{0}+g_{0} f_{0}^{\prime}\right] \chi_{1} } \\
= & {\left[\left(\frac{1}{k z}\right)^{4} g_{0} f_{0} \partial_{z} \chi_{1}\right]_{z=z_{1}}^{z=z_{2}}-M_{0} \int d z\left(\frac{1}{k z}\right)^{4}\left[f_{0}^{2}-g_{0}^{2}\right] \chi_{1} } \\
= & {\left[\left(\frac{1}{k z}\right)^{4} g_{0} f_{0} \partial_{z} \chi_{1}\right]_{z=z_{1}}^{z=z_{2}}-\frac{M_{0}}{g_{5} \sqrt{k}}\left(C_{R}-C_{L}\right), }
\end{aligned}
$$

where we have made use of the equation of motion of fermion field in the 5th dimension:

$$
\begin{aligned}
& f_{j}^{\prime}+M_{j} g_{j}-\frac{c+2}{z} f_{j}=0 \\
& g_{j}^{\prime}-M_{j} f_{j}+\frac{c-2}{z} g_{j}=0 .
\end{aligned}
$$


Here $M_{j}$ is the $4 \mathrm{D}$ mass of the $j$-th fermion KK mode, and $c$ is a bulk quark mass parameter, which doesn't appear in the interaction between fermion zero mode and $A_{5}^{(1)}$ :

$$
\int d^{4} x\left(-i \frac{M_{0}}{m_{K K}}\right)\left(C_{R}-C_{L}\right)\left(-\psi^{(0)} \chi^{(0)}+\bar{\chi}^{(0)} \bar{\psi}^{(0)}\right) A_{5}^{(1)} .
$$

The Feynman rules for the quark and first KK mode can be found in the appendix.

At this point, we have derived all the Feynman rules between the zero mode and the first KK mode that are uniquely determined by QCD gauge invariance, and the color representation of the KK mode. Vertices between the SM quark and first KK mode, though not fixed by gauge invariance, are also presented, since they are necessary for the process to happen. There exist other vertices which are not fixed by gauge invariance. For example, a vertex of $3 \mathrm{KK}$ gluon can be derived from the Lagrangian, with a coupling sensitive to the 5th dimension profile of the KK mode. Such couplings might not be small; instead they are strong coupling in many cases. However we choose to omit these interactions in our calculation for several reasons:

- These couplings are usually strong, the meaning of perturbative expansion is not clear.

- These couplings depend on the 5th dimension profile, thus are highly model dependent, and vary from model to model.

- The effects of these couplings can be calculated separately, if desired.

With the Feynman rules at hand, we are ready to explain the meaning of one-loop amplitudes for $q \bar{q} \rightarrow t \bar{t}$ in our calculation. These include the conventional SM QCD one-loop amplitudes, corrections of gluon self energy by loop of first KK mode, and the gluonic corrections to the LO process $q \bar{q} \rightarrow A_{\mu}^{(1)} \rightarrow t \bar{t}$. The amplitudes we consider have the features that they consist of a set of gauge invariant corrections, and are model independent (in the sense that only the mass and color representation of the first KK mode matter). The structure of IR divergence of these amplitudes resemble the SM QCD, and the IR divergences will be canceled when combining virtual corrections and real corrections. Thus all the low energy QCD effects are captured in our calculation, including the large threshold logarithms that usually dominate the NLO corrections [29]. The remaining diagrams that are not considered in this paper are both model dependent and IR finite. They can be calculated separately if needed. Similar consideration of calculating a subset of corrections can be found in ref. [30]. 


\section{ONE-LOOP HELICITY AMPLITUDE}

In this section we present the one-loop helicity amplitudes for $q \bar{q} \rightarrow t \bar{t}$, for both gluon induced and KK gluon induced processes. SM one-loop squared amplitudes for $t \bar{t}$ production are known for a long time [14-16, 31]. one-loop amplitudes with full helicity information are also known [32, 33]. We have re-derived the SM one-loop amplitude for $q \bar{q} \rightarrow t \bar{t}$ and found complete agreement with those in ref. [32]. Nevertheless we present them here for the sake of completeness.

\section{A. Convention}

Throughout our calculation, we adopt the Four-Dimensional Helicity (FDH) regularization scheme [34]. Therefore the gauge coupling is defined in the FDH scheme. The conventional $\overline{\mathrm{MS}}$ scheme gauge coupling can be obtained by a finite renormalization [35]

$$
\alpha_{s}^{\mathrm{FDH}}=\alpha_{s}^{\overline{\mathrm{MS}}}\left(1+\frac{\alpha_{s}^{\overline{\mathrm{MS}}}}{4 \pi}\right) .
$$

For simplicity, we do the calculation in 't Hooft-Feynman gauge, $\xi=1$. A common factor $C_{\epsilon}$ is omitted in all the result present below,

$$
C_{\epsilon}=\frac{1}{\Gamma(1-\epsilon)}(4 \pi)^{\epsilon} .
$$

Analytical continuation for the Mandelstam variables are defined as

$$
\begin{aligned}
& s \rightarrow s+i \varepsilon \\
& u \rightarrow u+i \varepsilon, \\
& t \rightarrow t+i \varepsilon .
\end{aligned}
$$

We use the modified spinor helicity method suitable for massive particles [36] in our calculation. A recent application of this method can be found in ref. [37]. As usual, massless spinor are denoted as

$$
\left|i^{ \pm}\right\rangle \equiv u_{ \pm}\left(k_{i}\right)=v_{\mp}\left(k_{i}\right), \quad\left\langle i^{ \pm}\right| \equiv \overline{u_{ \pm}\left(k_{i}\right)}=\overline{v_{\mp}\left(k_{i}\right)} .
$$

Massive momenta are written as sum of two massless momenta:

$$
p=p^{b}+\frac{M^{2}}{2 p \cdot \eta} \eta, \quad p^{2}=M^{2}, \quad\left(p^{b}\right)^{2}=\eta^{2}=0 .
$$


Massive spinor can then be written as

$$
\begin{aligned}
& u_{ \pm}\left(p, M ; \eta, p^{b}\right)=\frac{(\not p+M)\left|\eta^{\mp}\right\rangle}{\left\langle p^{b \pm} \mid \eta^{\mp}\right\rangle}, \quad \overline{u_{ \pm}}\left(p, M ; \eta, p^{b}\right)=\frac{\left\langle\eta^{\mp}\right|(\not p+M)}{\left\langle\eta^{\mp} \mid p^{p \pm}\right\rangle}, \\
& v_{ \pm}\left(p, M ; \eta, p^{b}\right)=\frac{(\not p-M)\left|\eta^{ \pm}\right\rangle}{\left\langle p^{p \mp} \mid \eta^{ \pm}\right\rangle}, \quad \overline{v_{ \pm}}\left(p, M ; \eta, p^{b}\right)=\frac{\left\langle\eta^{ \pm}\right|(\not p-M)}{\left\langle\eta^{ \pm} \mid p^{p \mp}\right\rangle},
\end{aligned}
$$

where $\eta$ is an arbitrary reference light-like momenta. The arbitrariness of $\eta$ can be utilized to change the helicity of massive spinor:

$$
\frac{\left\langle p^{b \mp} \mid \eta^{ \pm}\right\rangle}{M} \overline{u_{ \pm}}\left(p, M ; p^{b}, \eta\right)=\overline{u_{\mp}}\left(p, M ; \eta, p^{b}\right), \quad \frac{\left\langle p^{b \mp} \mid \eta^{ \pm}\right\rangle}{M} v_{ \pm}\left(p, M ; p^{b}, \eta\right)=v_{\mp}\left(p, M ; \eta, p^{b}\right) \text {. }
$$

Therefore we only give results for amplitudes with a definite helicity configuration of massive quark, $\lambda_{3}=+, \lambda_{4}=+$, where $\lambda_{3}$ and $\lambda_{4}$ are the helicity of $t$ and $\bar{t}$, respectively.

For the process we consider in this paper, the amplitudes can be factorized into the product of a color factor and color stripped spinor products, i.e., $\mathcal{M}=C \mathcal{A}$, where the color factor $C$ stands for some product of color matrixes. We will list below the color factor and spinor products respectively for each amplitude.

B. SM Helicity Amplitude for $q \bar{q} \rightarrow t \bar{t}$

1. Results for LO Diagrams

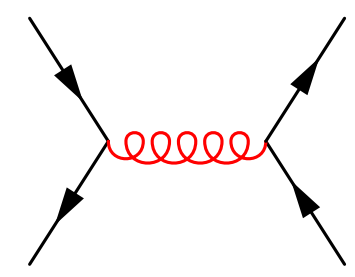

FIG. 1: SM tree graph for $q \bar{q} \rightarrow t \bar{t}$.

The LO amplitudes (Fig. 1) are straightforward to calculate. The color structure is

$$
\left(T^{a}\right)_{i_{2} i_{1}}\left(T^{a}\right)_{i_{3} i_{4}}=\frac{1}{2} \delta_{i_{3} i_{1}} \delta_{i_{2} i_{4}}-\frac{1}{2} \frac{1}{N_{c}} \delta_{i_{2} i_{1}} \delta_{i_{3} i_{4}} .
$$

The Lorentz part is made of two structures of spinor products

$$
\begin{aligned}
& \mathcal{A}_{\text {tree }}(+,-,+,+)=\frac{8 i \pi \alpha_{s} m_{t}}{s} \frac{\left\langle\eta_{4} 1\right\rangle\left\langle\eta_{3}|\mathbf{3}| 2\right]+\left\langle\eta_{3} 1\right\rangle\left\langle\eta_{4}|\mathbf{4}| 2\right]}{\left\langle 3^{b} \eta_{3}\right\rangle\left\langle\eta_{4} 4^{b}\right\rangle}, \\
& \mathcal{A}_{\text {tree }}(-,+,+,+)=\frac{8 i \pi \alpha_{s} m_{t}}{s} \frac{\left\langle\eta_{4} 2\right\rangle\left\langle\eta_{3}|\mathbf{3}| 1\right]+\left\langle\eta_{3} 2\right\rangle\left\langle\eta_{4}|\mathbf{4}| 1\right]}{\left\langle 3^{b} \eta_{3}\right\rangle\left\langle\eta_{4} 4^{b}\right\rangle},
\end{aligned}
$$


where the boldface momenta denote massive particle momentum vector. At the LO, there is only vector current coupling $\bar{\psi} \gamma^{\mu} \psi$ at the massive quark vertex. At the NLO, however, magnetic-moment coupling $\bar{\psi}\left(i \sigma^{\mu \nu} q_{\nu}\right) \psi /\left(2 m_{t}\right)$ is induced from loop diagram. Here we have defined $q=p_{3}+p_{4}$. For completeness we also list tree amplitudes for magnetic-moment interaction as follows:

$$
\begin{aligned}
& \mathcal{A}_{\text {tree }}^{(m)}(+,-,+,+)=-\frac{4 i \pi \alpha_{s}}{s m_{t}} \frac{m_{t}^{2}[21]\left\langle\eta_{3} 1\right\rangle\left\langle\eta_{4} 1\right\rangle+\langle 12\rangle\left\langle\eta_{3}|\mathbf{3}| 2\right]\left\langle\eta_{4}|\mathbf{4}| 2\right]}{\left\langle 3^{b} \eta_{3}\right\rangle\left\langle\eta_{4} 4^{b}\right\rangle}, \\
& \mathcal{A}_{\text {tree }}^{(m)}(-,+,+,+)=\frac{4 i \pi \alpha_{s}}{s m_{t}} \frac{m_{t}^{2}[21]\left\langle\eta_{3} 2\right\rangle\left\langle\eta_{4} 2\right\rangle+\langle 12\rangle\left\langle\eta_{3}|\mathbf{3}| 1\right]\left\langle\eta_{4}|\mathbf{4}| 1\right]}{\left\langle 3^{b} \eta_{3}\right\rangle\left\langle\eta_{4} 4^{b}\right\rangle}
\end{aligned}
$$

\section{Results for Self-energy Diagrams}


FIG. 2: SM one-loop self energy graphs for $q \bar{q} \rightarrow t \bar{t}$.

The amplitudes for self-energy diagrams (Fig. 2) are proportional to the tree amplitudes. The color structure is identical to that of tree amplitudes. The Lorentz part is UV-divergent. The contributions from $n_{f}$ massless quark flavors, the massive top quark and the gluonic 
self-interactions are found to be

$$
\begin{aligned}
\mathcal{A}_{s f}^{q}\left(\lambda_{1}, \lambda_{2}, \lambda_{3}, \lambda_{4}\right)= & \mathcal{A}_{\text {tree }}\left(\lambda_{1}, \lambda_{2}, \lambda_{3}, \lambda_{4}\right) \frac{\alpha_{s}}{6 \pi} n_{f}\left\{-\frac{1}{\epsilon_{\mathrm{UV}}}+\ln \left(-\frac{s}{\mu^{2}}\right)-\frac{5}{3}\right\}, \\
\mathcal{A}_{s f}^{t}\left(\lambda_{1}, \lambda_{2}, \lambda_{3}, \lambda_{4}\right)= & \mathcal{A}_{\text {tree }}\left(\lambda_{1}, \lambda_{2}, \lambda_{3}, \lambda_{4}\right) \frac{\alpha_{s}}{6 \pi}\left\{-\frac{1}{\epsilon_{\mathrm{UV}}}+\frac{2 m_{t}^{2}}{s}\left(\beta \ln \left(\frac{\beta+1}{\beta-1}\right)-2\right)\right. \\
& \left.+\beta \ln \left(\frac{\beta+1}{\beta-1}\right)+\ln \left(\frac{m_{t}^{2}}{\mu^{2}}\right)-\frac{5}{3}\right\} \\
\mathcal{A}_{s f}^{g}\left(\lambda_{1}, \lambda_{2}, \lambda_{3}, \lambda_{4}\right)= & \mathcal{A}_{\text {tree }}\left(\lambda_{1}, \lambda_{2}, \lambda_{3}, \lambda_{4}\right) \frac{\alpha_{s}}{\pi}\left\{\frac{5}{4 \epsilon_{\mathrm{UV}}}-\frac{5}{4} \ln \left(-\frac{s}{\mu^{2}}\right)+\frac{7}{3}\right\} .
\end{aligned}
$$

For $n_{f}=5$ massless quark flavors, the total self-energy diagram amplitude is simply

$$
\begin{aligned}
\mathcal{A}_{s f}\left(\lambda_{1}, \lambda_{2}, \lambda_{3}, \lambda_{4}\right)= & \mathcal{A}_{\text {tree }}\left(\lambda_{1}, \lambda_{2}, \lambda_{3}, \lambda_{4}\right) \frac{\alpha_{s}}{12 \pi}\left\{\frac{3}{\epsilon_{\mathrm{UV}}}-\frac{8 m_{t}^{2}}{s}-5 \ln \left(-\frac{s}{\mu^{2}}\right)+2 \ln \left(\frac{m_{t}^{2}}{\mu^{2}}\right)\right. \\
& \left.+2\left(\frac{2 m_{t}^{2}}{s}+1\right) \beta \ln \left(\frac{\beta+1}{\beta-1}\right)+8\right\} .
\end{aligned}
$$

At one-loop level, the massive KK-gluon also enters the gluon propagator via gauge in-
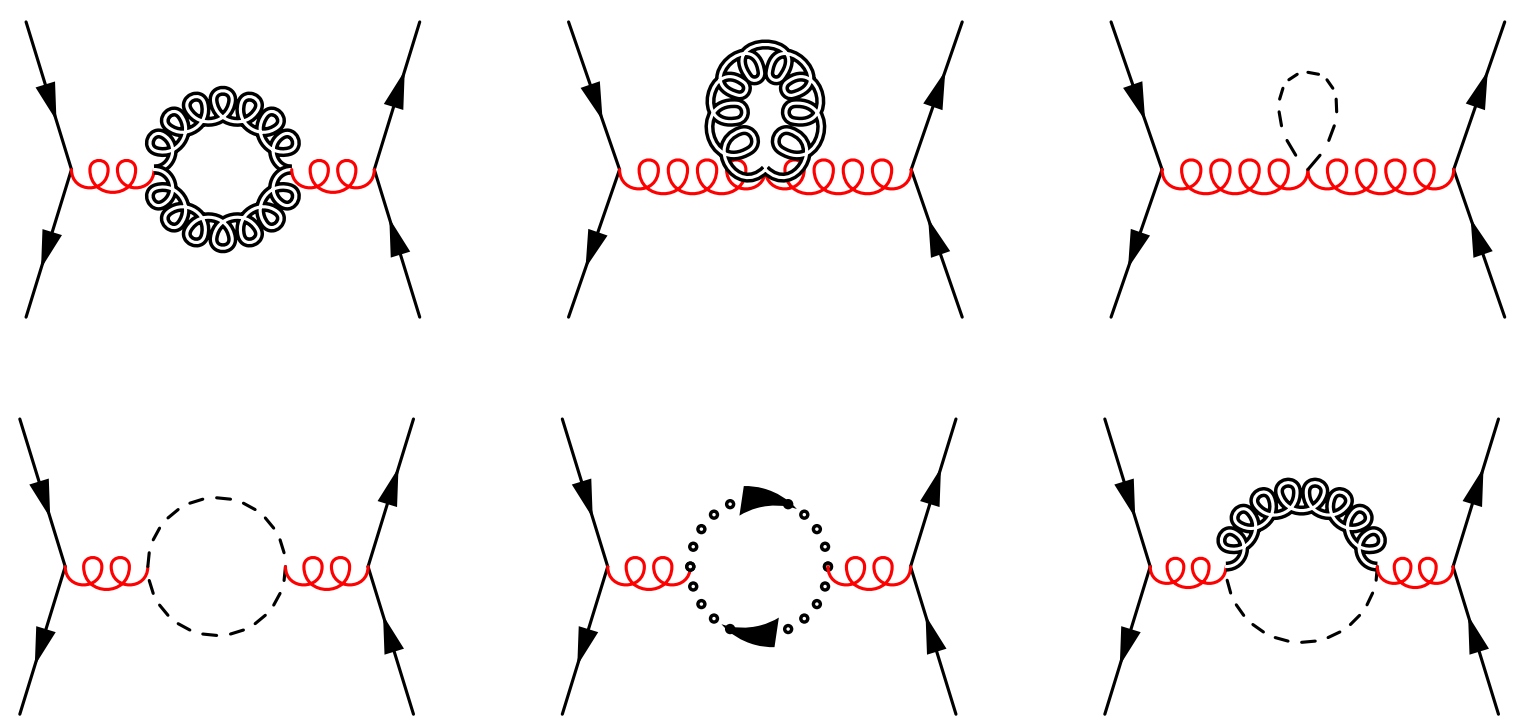

FIG. 3: KK gluon induced one-loop gluon self energy graph for $q \bar{q} \rightarrow t \bar{t}$.

teractions with the gluon, and hence contributes to the gluon self-energy function. These diagrams (Fig. 3in) give arise to an additional amplitude

$$
\begin{aligned}
\mathcal{A}_{s f}^{K K}\left(\lambda_{1}, \lambda_{2}, \lambda_{3}, \lambda_{4}\right)= & \mathcal{A}_{\text {tree }}\left(\lambda_{1}, \lambda_{2}, \lambda_{3}, \lambda_{4}\right) \frac{\alpha_{s}}{8 \pi}\left\{\frac{9}{\epsilon_{\mathrm{UV}}}-9 \ln \left(\frac{m_{K K}^{2}}{\mu^{2}}\right)+8\left(\frac{3 m_{K K}^{2}}{s}+2\right)\right. \\
& \left.-3\left(\frac{4 m_{K K}^{2}}{s}+3\right) \tilde{\beta} \ln \left(\frac{\tilde{\beta}+1}{\tilde{\beta}-1}\right)\right\} .
\end{aligned}
$$


Here we have defined $\tilde{\beta}=\sqrt{1-4 m_{K K}^{2} / s}$.

The gluon wave-function renormalization constants enters the renormalization constants of the strong coupling. We renormalize the massless quark loops and gluonic loops in the $\overline{\mathrm{MS}}$ scheme, while for the massive top quark loop and KK gluon loop, on-shell scheme is adopted. Thus, massive particles are decoupled from the running of the strong coupling constant. Explicitly, various contributions to the gluon wave-function renormalization constants are

$$
\delta Z_{g}=\delta Z_{g}^{(q), \overline{\mathrm{MS}}}+\delta Z_{g}^{(t), \mathrm{OS}}+\delta Z_{g}^{(g), \overline{\mathrm{MS}}}+\delta Z_{g}^{(K K), \mathrm{OS}}
$$

where

$$
\begin{aligned}
\delta Z_{g}^{(q), \overline{\mathrm{MS}}} & =\frac{\alpha_{s}}{\pi} n_{f}\left\{-\frac{1}{6 \epsilon_{\mathrm{UV}}}\right\}, \\
\delta Z_{g}^{(t), \mathrm{OS}} & =\frac{\alpha_{s}}{\pi}\left\{-\frac{1}{6 \epsilon_{\mathrm{UV}}}+\frac{1}{6} \ln \left(\frac{m_{t}^{2}}{\mu^{2}}\right)\right\}, \\
\delta Z_{g}^{(g), \overline{\mathrm{MS}}} & =\frac{\alpha_{s}}{\pi}\left\{\frac{5}{4 \epsilon_{\mathrm{UV}}}\right\}, \\
\delta Z_{g}^{(K K), \mathrm{OS}} & =\frac{\alpha_{s}}{\pi}\left\{\frac{9}{8 \epsilon_{\mathrm{UV}}}-\frac{9}{8} \ln \left(\frac{m_{K K}^{2}}{\mu^{2}}\right)\right\} .
\end{aligned}
$$

The corresponding counter-term diagram which renders the self-energy correction finite is

$$
\begin{aligned}
\mathcal{A}_{s f}^{C T}\left(\lambda_{1}, \lambda_{2}, \lambda_{3}, \lambda_{4}\right) & =\mathcal{A}_{\text {tree }}\left(\lambda_{1}, \lambda_{2}, \lambda_{3}, \lambda_{4}\right) \times\left(-\delta Z_{g}\right) \\
& =\mathcal{A}_{\text {tree }}\left(\lambda_{1}, \lambda_{2}, \lambda_{3}, \lambda_{4}\right) \frac{\alpha_{s}}{\pi}\left\{-\frac{11}{8 \epsilon_{\mathrm{UV}}}-\frac{1}{6} \ln \left(\frac{m_{t}^{2}}{\mu^{2}}\right)+\frac{9}{8} \ln \left(\frac{m_{K K}^{2}}{\mu^{2}}\right)\right\} .
\end{aligned}
$$

\section{Results for Triangle Diagrams}

The color structure for the triangle diagram (Fig 4) is the same as the tree amplitude. The Lorentz part of the amplitude can be divided into two parts. One is the vector current coupling induced part, which is UV- and IR- divergent at one-loop level. The other is the magnetic-moment type interaction induced part, which is free of divergence. Accordingly, we introduce two form factors $F_{1,2}\left(m_{t}^{2}, s\right)$ and write the triangle diagram contribution as

$$
\mathcal{A}_{v t}\left(\lambda_{1}, \lambda_{2}, \lambda_{3}, \lambda_{4}\right)=\mathcal{A}_{\text {tree }}\left(\lambda_{1}, \lambda_{2}, \lambda_{3}, \lambda_{4}\right) F_{1}\left(m_{t}^{2}, s\right)+\mathcal{A}_{\text {tree }}^{(m)}\left(\lambda_{1}, \lambda_{2}, \lambda_{3}, \lambda_{4}\right) F_{2}\left(m_{t}^{2}, s\right)
$$





FIG. 4: SM one-loop triangle graphs for $q \bar{q} \rightarrow t \bar{t}$.

The one-loop triangle diagram of massless quark contributes only to the vector current form factor $F_{1}\left(m_{t}^{2}, s\right)$ :

$$
\begin{aligned}
\mathcal{A}_{v t}^{q}\left(\lambda_{1}, \lambda_{2}, \lambda_{3}, \lambda_{4}\right)= & \mathcal{A}_{\text {tree }}\left(\lambda_{1}, \lambda_{2}, \lambda_{3}, \lambda_{4}\right) \frac{\alpha_{s}}{4 \pi}\left\{\frac{13}{3 \epsilon_{\mathrm{UV}}}+\frac{1}{3 \epsilon_{\mathrm{IR}}^{2}}-\frac{1}{\epsilon_{\mathrm{IR}}}\left(\frac{1}{3} \ln \left(-\frac{s}{\mu^{2}}\right)+\frac{16}{3}\right)\right. \\
& \left.+\frac{1}{6} \ln ^{2}\left(-\frac{s}{\mu^{2}}\right)+\ln \left(-\frac{s}{\mu^{2}}\right)-\frac{1}{3}\right\} .
\end{aligned}
$$


The one-loop triangle diagram of massive quark contributes to both $F_{1}\left(m_{t}^{2}, s\right)$ and $F_{2}\left(m_{t}^{2}, s\right)$ :

$$
\begin{aligned}
& \mathcal{A}_{v t}^{t}\left(\lambda_{1}, \lambda_{2}, \lambda_{3}, \lambda_{4}\right)=\mathcal{A}_{\text {tree }}\left(\lambda_{1}, \lambda_{2}, \lambda_{3}, \lambda_{4}\right) \frac{\alpha_{s}}{4 \pi}\left\{\frac{13}{3 \epsilon_{\mathrm{UV}}}+\frac{1}{\epsilon_{\mathrm{IR}}} \frac{2 m_{t}^{2}-s}{3 s \beta} \ln \left(\frac{\beta+1}{\beta-1}\right)\right. \\
& +\frac{1}{6 s \beta s_{1}^{2}}\left[9 \beta \ln \left(-\frac{s}{\mu^{2}}\right)\left(s-16 m_{t}^{2}\right) s^{2}+36 \mathrm{Li}_{2}\left(\frac{1}{1-\beta}\right) m_{t}^{2}\left(8 m_{t}^{2}+s\right) s\right. \\
& -72 \mathrm{Li}_{2}(1-\beta) m_{t}^{2}\left(8 m_{t}^{2}+s\right) s+36 \mathrm{Li}_{2}\left(\frac{1}{\beta+1}\right) m_{t}^{2}\left(8 m_{t}^{2}+s\right) s \\
& -36 \mathrm{Li}_{2}\left(\frac{\beta+1}{1-\beta}\right) m_{t}^{2}\left(8 m_{t}^{2}+s\right) s-18 \mathrm{Li}_{2}\left(\frac{s}{4 m_{t}^{2}}\right) m_{t}^{2}\left(8 m_{t}^{2}+s\right) s \\
& +18 \mathrm{Li}_{2}\left(\frac{4 m_{t}^{2}}{s}\right) m_{t}^{2}\left(8 m_{t}^{2}+s\right) s-\beta \ln \left(\frac{m_{t}^{2}}{\mu^{2}}\right)\left(416 m_{t}^{4}-352 s m_{t}^{2}+35 s^{2}\right) s \\
& +2\left(3 \pi^{2}\left(8 m_{t}^{2}+s\right) m_{t}^{2}+\beta\left(352 m_{t}^{4}-212 s m_{t}^{2}+31 s^{2}\right)\right) s-2 \mathrm{Li}_{2}\left(\frac{\beta-1}{2 \beta}\right)\left(2 m_{t}^{2}-s\right) s_{1}^{2} \\
& +2 \mathrm{Li}_{2}\left(\frac{\beta+1}{2 \beta}\right)\left(2 m_{t}^{2}-s\right) s_{1}^{2}+\ln \left(\frac{\beta+1}{\beta-1}\right)\left(-s\left(3 s-8 m_{t}^{2}\right) s_{1} \beta^{2}\right. \\
& \left.\left.\left.-2 \ln (\beta)\left(2 m_{t}^{2}-s\right) s_{1}^{2}-2 \ln \left(-\frac{s}{\mu^{2}}\right)\left(2 m_{t}^{2}-s\right) s_{1}^{2}-\ln \left(-\frac{m_{t}^{2}}{s}\right)\left(2 m_{t}^{2}-s\right) s_{1}^{2}\right)\right]\right\} \\
& +\mathcal{A}_{t r e e}^{(m)}\left(\lambda_{1}, \lambda_{2}, \lambda_{3}, \lambda_{4}\right) \frac{\alpha_{s}}{6 \pi} \frac{m_{t}^{2}}{\beta s_{1}^{2}}\left\{\ln \left(\frac{\beta+1}{\beta-1}\right) s_{1} \beta^{2}+9 \ln \left(-\frac{s}{\mu^{2}}\right)\left(8 m_{t}^{2}+s\right) \beta\right. \\
& -9 \ln \left(\frac{m_{t}^{2}}{\mu^{2}}\right)\left(8 m_{t}^{2}+s\right) \beta-108 \mathrm{Li}_{2}\left(\frac{1}{1-\beta}\right) m_{t}^{2}+216 \mathrm{Li}_{2}(1-\beta) m_{t}^{2}-108 \mathrm{Li}_{2}\left(\frac{1}{\beta+1}\right) m_{t}^{2} \\
& \left.+108 \mathrm{Li}_{2}\left(\frac{\beta+1}{1-\beta}\right) m_{t}^{2}+54 \mathrm{Li}_{2}\left(\frac{s}{4 m_{t}^{2}}\right) m_{t}^{2}-54 \mathrm{Li}_{2}\left(\frac{4 m_{t}^{2}}{s}\right) m_{t}^{2}-18\left(\pi^{2} m_{t}^{2}+\beta s_{1}\right)\right\}
\end{aligned}
$$

The renormalization constant of the strong coupling $g_{s}$ is given by

$$
\delta Z_{g_{s}}=-\delta Z_{\Gamma}^{\overline{\mathrm{MS}}}-\delta Z_{q}^{\overline{\mathrm{MS}}}-\frac{1}{2} \delta Z_{g}^{\overline{\mathrm{MS}}}
$$

where $\delta Z_{\Gamma}^{\overline{\mathrm{MS}}}$ is the UV-divergent part of the one-loop vertex function:

$$
\delta Z_{\Gamma}^{\overline{M S}}=\frac{\alpha_{s}}{\pi} \frac{13}{12 \epsilon_{\mathrm{UV}}}
$$

and $\delta Z_{q}^{\overline{\mathrm{MS}}}$ is just the UV-divergent part of the on-shell wave-function renormalization constant for massless quark. The on-shell wave-function renormalization constants for massless and massive quark are

$$
\begin{aligned}
\delta Z_{q}^{\mathrm{OS}} & =-\frac{\alpha_{s}}{3 \pi}\left\{\frac{1}{\epsilon_{\mathrm{UV}}}-\frac{1}{\epsilon_{\mathrm{IR}}}\right\} \\
\delta Z_{t}^{\mathrm{OS}} & =\frac{\alpha_{s}}{3 \pi}\left\{-\frac{1}{\epsilon_{\mathrm{UV}}}-\frac{2}{\epsilon_{\mathrm{IR}}}+3 \ln \left(\frac{m_{t}^{2}}{\mu^{2}}\right)-5\right\}
\end{aligned}
$$


The counter-term contributions that render both the massless quark vertex and the massive quark vertex to be UV-finite, respectively, are:

$$
\begin{aligned}
\mathcal{A}_{v t}^{q, C T}\left(\lambda_{1}, \lambda_{2}, \lambda_{3}, \lambda_{4}\right) & =\mathcal{A}_{\text {tree }}\left(\lambda_{1}, \lambda_{2}, \lambda_{3}, \lambda_{4}\right) \times\left(-\delta Z_{\Gamma}^{\overline{\mathrm{MS}}}-\delta Z_{q}^{\overline{\mathrm{MS}}}+\delta Z_{q}^{\mathrm{OS}}\right) \\
& =\mathcal{A}_{\text {tree }}\left(\lambda_{1}, \lambda_{2}, \lambda_{3}, \lambda_{4}\right) \frac{\alpha_{s}}{\pi}\left\{-\frac{13}{12 \epsilon_{\mathrm{UV}}}+\frac{1}{3 \epsilon_{\mathrm{IR}}}\right\} \\
\mathcal{A}_{v t}^{t, C T}\left(\lambda_{1}, \lambda_{2}, \lambda_{3}, \lambda_{4}\right) & =\mathcal{A}_{\text {tree }}\left(\lambda_{1}, \lambda_{2}, \lambda_{3}, \lambda_{4}\right) \times\left(-\delta Z_{\Gamma}^{\overline{\mathrm{MS}}}-\delta Z_{t}^{\overline{\mathrm{MS}}}+\delta Z_{t}^{\mathrm{OS}}\right) \\
& =\mathcal{A}_{\text {tree }}\left(\lambda_{1}, \lambda_{2}, \lambda_{3}, \lambda_{4}\right) \frac{\alpha_{s}}{\pi}\left\{-\frac{13}{12 \epsilon_{\mathrm{UV}}}-\frac{2}{3 \epsilon_{\mathrm{IR}}}+\ln \left(\frac{m_{t}^{2}}{\mu^{2}}\right)-\frac{5}{3}\right\}
\end{aligned}
$$

4. Results for Box Diagrams
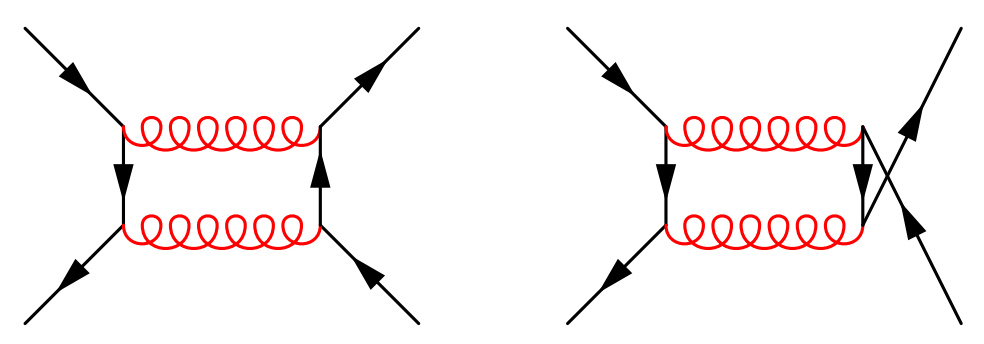

FIG. 5: SM regular and cross box diagrams.

For each of the two helicity configurations for massless quarks, the Lorentz parts of the box diagram (Fig (5) amplitudes can be reduced to contain only 4 independent structure of spinor product. For the regular box diagram, which is proportional to the color structure

$$
\left(T^{a} T^{b}\right)_{i_{2} i_{1}}\left(T^{b} T^{a}\right)_{i_{3} i_{4}}=\frac{1}{4}\left(N_{c}-\frac{2}{N_{c}}\right) \delta_{i_{3} i_{1}} \delta_{i_{2} i_{4}}+\frac{1}{4} \frac{1}{N_{c}^{2}} \delta_{i_{2} i_{1}} \delta_{i_{3} i_{4}}
$$

we have found

$$
\begin{aligned}
\mathcal{A}_{b 1}(+,-,+,+)= & \frac{4 i \alpha_{s}^{2} m_{t}}{\left\langle 3^{b} \eta_{3}\right\rangle\left\langle\eta_{4} 4^{b}\right\rangle}\left\{B_{1}\left\langle\eta_{4} 1\right\rangle\left\langle\eta_{3}|\mathbf{3}| 2\right]+B_{2}\left\langle\eta_{3} 1\right\rangle\left\langle\eta_{4}|\mathbf{4}| 2\right]\right. \\
& \left.+B_{3}\left[m_{t}^{2}[21]\left\langle\eta_{3} 1\right\rangle\left\langle\eta_{4} 1\right\rangle+\langle 12\rangle\left\langle\eta_{3}|\mathbf{3}| 2\right]\left\langle\eta_{4}|\mathbf{4}| 2\right]\right]\right\}, \\
\mathcal{A}_{b 1}(-,+,+,+)= & \frac{4 i \alpha_{s}^{2} m_{t}}{\left\langle 3^{b} \eta_{3}\right\rangle\left\langle\eta_{4} 4^{b}\right\rangle}\left\{B_{2}\left\langle\eta_{4} 2\right\rangle\left\langle\eta_{3}|\mathbf{3}| 1\right]+B_{1}\left\langle\eta_{3} 2\right\rangle\left\langle\eta_{4}|\mathbf{4}| 1\right]\right. \\
& \left.-B_{3}\left[m_{t}^{2}[21]\left\langle\eta_{3} 2\right\rangle\left\langle\eta_{4} 2\right\rangle+\langle 12\rangle\left\langle\eta_{3}|\mathbf{3}| 1\right]\left\langle\eta_{4}|\mathbf{4}| 1\right]\right]\right\},
\end{aligned}
$$

where $B_{i}, i=1,2,3$, are auxiliary functions that depend on $m_{t}^{2}$ and the Mandelstam variables $s, t, u$. These functions can be expressed in terms of the Passarino-Veltman one-loop 
functions

$$
\begin{aligned}
B_{1}= & 2 D_{00}-t\left(D_{0}+D_{1}+D_{3}+D_{13}\right)+m_{t}^{2}\left(D_{0}+2 D_{1}+D_{3}+D_{11}+D_{13}\right), \\
B_{2}= & 8 D_{00}-t\left(D_{0}+D_{1}+D_{3}+2 D_{13}\right)+m_{t}^{2}\left(D_{0}+2 D_{1}+D_{3}+3 D_{11}+2 D_{13}\right), \\
& +2 s\left(D_{2}+D_{12}+D_{22}+D_{23}\right), \\
B_{3}= & -D_{12},
\end{aligned}
$$

where $D_{i}, D_{i j}$ are defined as

$$
\begin{aligned}
D_{i} & =\operatorname{PaVe}\left[i,\left\{m_{t}^{2}, m_{t}^{2}, 0,0, s, t\right\},\left\{0, m_{t}^{2}, 0,0\right\}\right] \\
D_{i j} & =\operatorname{PaVe}\left[i, j,\left\{m_{t}^{2}, m_{t}^{2}, 0,0, s, t\right\},\left\{0, m_{t}^{2}, 0,0\right\}\right] .
\end{aligned}
$$

We can define crossed auxiliary functions by exchange $t \longleftrightarrow u, \tilde{B}_{i}=-B_{i}(t \longleftrightarrow u)$. The amplitudes for the crossed box diagram, which is proportional to the color structure

$$
\left(T^{a} T^{b}\right)_{i_{2} i_{1}}\left(T^{a} T^{b}\right)_{i_{3} i_{4}}=-\frac{1}{4} \frac{2}{N_{c}} \delta_{i_{3} i_{1}} \delta_{i_{2} i_{4}}+\frac{1}{4}\left(1+\frac{1}{N_{c}^{2}}\right) \delta_{i_{2} i_{1}} \delta_{i_{3} i_{4}}
$$

are

$$
\begin{aligned}
\mathcal{A}_{b 2}(+,-,+,+)= & \frac{4 i \alpha_{s}^{2} m_{t}}{\left\langle 3^{b} \eta_{3}\right\rangle\left\langle\eta_{4} 4^{b}\right\rangle}\left\{\tilde{B}_{2}\left\langle\eta_{4} 1\right\rangle\left\langle\eta_{3}|\mathbf{3}| 2\right]+\tilde{B}_{1}\left\langle\eta_{3} 1\right\rangle\left\langle\eta_{4}|\mathbf{4}| 2\right]\right. \\
& \left.+\tilde{B}_{3}\left[m_{t}^{2}[21]\left\langle\eta_{3} 1\right\rangle\left\langle\eta_{4} 1\right\rangle+\langle 12\rangle\left\langle\eta_{3}|\mathbf{3}| 2\right]\left\langle\eta_{4}|\mathbf{4}| 2\right]\right]\right\}, \\
\mathcal{A}_{b 2}(-,+,+,+)= & \frac{4 i \alpha_{s}^{2} m_{t}}{\left\langle 3^{b} \eta_{3}\right\rangle\left\langle\eta_{4} 4^{b}\right\rangle}\left\{\tilde{B}_{1}\left\langle\eta_{4} 2\right\rangle\left\langle\eta_{3}|\mathbf{3}| 1\right]+\tilde{B}_{2}\left\langle\eta_{3} 2\right\rangle\left\langle\eta_{4}|\mathbf{4}| 1\right]\right. \\
& \left.-\tilde{B}_{3}\left[m_{t}^{2}[21]\left\langle\eta_{3} 2\right\rangle\left\langle\eta_{4} 2\right\rangle+\langle 12\rangle\left\langle\eta_{3}|\mathbf{3}| 1\right]\left\langle\eta_{4}|\mathbf{4}| 1\right]\right]\right\} .
\end{aligned}
$$

Next we give explicit expressions for $B_{i}$, suitable for general complex arguments. First we introduce some notations

$$
s_{1}=s-4 m_{t}^{2}, \quad t_{1}=m_{t}^{2}-t, \quad u_{1}=m_{t}^{2}-u, \quad K=m_{t}^{4}-t u, \quad \beta=\sqrt{1-4 m_{t}^{2} / s}
$$

$B_{3}$ is finite

$$
\begin{aligned}
B_{3}= & -\frac{1}{K^{2} s_{1} s \beta}\left\{\operatorname{Li}_{2}\left(\frac{1}{1-\beta}\right)-2 \operatorname{Li}_{2}(1-\beta)+\operatorname{Li}_{2}\left(\frac{1}{\beta+1}\right)-\operatorname{Li}_{2}\left(\frac{\beta+1}{1-\beta}\right)+\frac{1}{2} \operatorname{Li}_{2}\left(\frac{4 m_{t}^{2}}{s}\right)\right. \\
& \left.-\frac{1}{2} \operatorname{Li}_{2}\left(\frac{s}{4 m_{t}^{2}}\right)+\frac{\pi^{2}}{6}\right\} C_{1}-\frac{1}{12 K^{2} s_{1}}\left\{\left(-3 s_{1} \ln ^{2}\left(-\frac{s}{\mu^{2}}\right) t_{1}^{2}-6 s_{1} \ln ^{2}\left(\frac{t_{1}}{\mu^{2}}\right) t_{1}^{2}\right.\right. \\
& +3 s_{1} \ln ^{2}\left(\frac{m_{t}^{2}}{\mu^{2}}\right) t_{1}^{2}-4 \pi^{2} s_{1} t_{1}^{2}+12 s_{1} \operatorname{Li}_{2}\left(-\frac{t}{t_{1}}\right) t_{1}^{2}+12 K s \ln \left(\frac{t_{1}}{\mu^{2}}\right) \\
& +\ln \left(-\frac{s}{\mu^{2}}\right)\left(12 s_{1} \ln \left(\frac{t_{1}}{\mu^{2}}\right) t_{1}^{2}+12 K\left(m_{t}^{2}+t\right)\right) \\
& \left.+\ln \left(\frac{m_{t}^{2}}{\mu^{2}}\right)\left(12 K\left(m_{t}^{2}+u\right)-6 s_{1} t_{1}^{2} \ln \left(-\frac{s}{\mu^{2}}\right)\right)\right\}
\end{aligned}
$$


where the coefficient $C_{1}$ is a polynomial of $m_{t}^{2}, s, t, u$

$$
C_{1}=4 m_{t}^{8}-6 s m_{t}^{6}-12 t m_{t}^{6}+s^{2} m_{t}^{4}+12 t^{2} m_{t}^{4}+8 s t m_{t}^{4}-4 t^{3} m_{t}^{2}-2 s t^{2} m_{t}^{2}-2 s^{2} t m_{t}^{2}-s^{2} t^{2} .
$$

The other two functions $B_{1}, B_{2}$ have the same IR-divergent part so that divergences are proportional to the tree amplitudes

$$
B_{1,2}=B_{1,2}^{f i n}+\frac{1}{s}\left\{-\frac{1}{\epsilon_{\mathrm{IR}}^{2}}+\frac{1}{\epsilon_{\mathrm{IR}}}\left(2 \ln \left(\frac{t_{1}}{\mu^{2}}\right)-\ln \left(\frac{m_{t}^{2}}{\mu^{2}}\right)\right)\right\},
$$

where $B_{1,2}^{f i n}$ are the finite parts, respectively. We also give explicit expressions for both finite parts. For $B_{2}^{\text {fin }}$, we define coefficients

$$
\begin{aligned}
C_{2}= & 4 m_{t}^{8}-7 t m_{t}^{6}-3 u m_{t}^{6}+5 t^{2} m_{t}^{4}+u^{2} m_{t}^{4}+4 t u m_{t}^{4}-t^{3} m_{t}^{2}-2 t u^{2} m_{t}^{2}-3 t^{2} u m_{t}^{2}+2 t^{2} u^{2}, \\
C_{3}= & m_{t}^{4}-3 t m_{t}^{2}-u m_{t}^{2}+t^{2}+2 t u \\
C_{4}= & 2 t m_{t}^{8}-6 u m_{t}^{8}-7 t^{2} m_{t}^{6}+u^{2} m_{t}^{6}+6 t u m_{t}^{6}-t^{3} m_{t}^{4}+u^{3} m_{t}^{4}+5 t u^{2} m_{t}^{4}+3 t^{2} u m_{t}^{4}+t^{4} m_{t}^{2} \\
& -2 t u^{3} m_{t}^{2}-3 t^{2} u^{2} m_{t}^{2}+4 t^{3} u m_{t}^{2}-2 t^{2} u^{3}-2 t^{3} u^{2}, \\
C_{5}= & 7 m_{t}^{8}-14 t m_{t}^{6}-6 u m_{t}^{6}+10 t^{2} m_{t}^{4}+2 u^{2} m_{t}^{4}+10 t u m_{t}^{4}-2 t^{3} m_{t}^{2}-4 t u^{2} m_{t}^{2}-6 t^{2} u m_{t}^{2}+3 t^{2} u^{2}, \\
C_{6}= & 2 m_{t}^{4}-t m_{t}^{2}+u m_{t}^{2}-2 t u .
\end{aligned}
$$

We have

$$
\begin{aligned}
B_{2}^{f i n}= & \frac{C_{2}}{4 K^{2} s} \ln \left(-\frac{s}{\mu^{2}}\right)\left(\ln \left(-\frac{s}{\mu^{2}}\right)-4 \ln \left(\frac{t_{1}}{\mu^{2}}\right)+2 \ln \left(\frac{m_{t}^{2}}{\mu^{2}}\right)\right)+\frac{C_{3} m_{t}^{2}}{4 K^{2}}\left(2 \ln ^{2}\left(\frac{t_{1}}{\mu^{2}}\right)\right. \\
& \left.-\ln ^{2}\left(\frac{m_{t}^{2}}{\mu^{2}}\right)-4 \operatorname{Li}_{2}\left(-\frac{t}{t_{1}}\right)\right)+\frac{C_{4}}{K^{2} s_{1} s \beta}\left(\operatorname{Li}_{2}\left(\frac{1}{1-\beta}\right)-2 \operatorname{Li}_{2}(1-\beta)+\operatorname{Li}_{2}\left(\frac{1}{\beta+1}\right)\right. \\
& \left.-\operatorname{Li}_{2}\left(\frac{\beta+1}{1-\beta}\right)+\frac{1}{2} \operatorname{Li}_{2}\left(\frac{4 m_{t}^{2}}{s}\right)-\frac{1}{2} \operatorname{Li}_{2}\left(\frac{s}{4 m_{t}^{2}}\right)+\frac{\pi^{2}}{6}\right)+\frac{\pi^{2} C_{5}}{6 K^{2} s}+\frac{C_{6} \ln \left(\frac{m_{t}^{2}}{\mu^{2}}\right) m_{t}^{2}}{K s_{1} t} \\
& +\frac{m_{t}^{2}}{K s_{1} t}\left(t(u-t) \ln \left(-\frac{s}{\mu^{2}}\right)+s_{1} t_{1} \ln \left(\frac{t_{1}}{\mu^{2}}\right)\right) .
\end{aligned}
$$

For $B_{1}^{f i n}$, we define another set of coefficients

$$
\begin{aligned}
C_{7}= & 4 m_{t}^{8}-9 t m_{t}^{6}-u m_{t}^{6}+10 t^{2} m_{t}^{4}-5 t^{3} m_{t}^{2}-t^{2} u m_{t}^{2}+t^{4}+t^{2} u^{2} \\
C_{8}= & m_{t}^{6}-4 t m_{t}^{4}+3 t^{2} m_{t}^{2}-t^{3}+t^{2} u \\
C_{9}= & 6 s m_{t}^{8}+8 t m_{t}^{8}-s^{2} m_{t}^{6}-24 t^{2} m_{t}^{6}-12 s t m_{t}^{6}+24 t^{3} m_{t}^{4}+16 s t^{2} m_{t}^{4}+4 s^{2} t m_{t}^{4}-8 t^{4} m_{t}^{2} \\
& -12 s t^{3} m_{t}^{2}-5 s^{2} t^{2} m_{t}^{2}+2 s t^{4}+2 s^{2} t^{3}+s^{3} t^{2} \\
C_{10}= & 7 m_{t}^{8}-18 t m_{t}^{6}-2 u m_{t}^{6}+20 t^{2} m_{t}^{4}+2 t u m_{t}^{4}-10 t^{3} m_{t}^{2}-2 t^{2} u m_{t}^{2}+2 t^{4}+t^{2} u^{2} \\
C_{11}= & 2 m_{t}^{4}-t^{2}-t u .
\end{aligned}
$$


We have

$$
\begin{aligned}
B_{1}^{f i n}= & \frac{C_{7}}{4 K^{2} s} \ln \left(-\frac{s}{\mu^{2}}\right)\left(\ln \left(-\frac{s}{\mu^{2}}\right)-4 \ln \left(\frac{t_{1}}{\mu^{2}}\right)+2 \ln \left(\frac{m_{t}^{2}}{\mu^{2}}\right)\right)-\frac{C_{8}}{4 K^{2}}\left(-2 \ln ^{2}\left(\frac{t_{1}}{\mu^{2}}\right)\right. \\
& \left.+\ln ^{2}\left(\frac{m_{t}^{2}}{\mu^{2}}\right)+4 \operatorname{Li}_{2}\left(-\frac{t}{t_{1}}\right)\right)+\frac{C_{9}}{K^{2} s_{1} s \beta}\left(\operatorname{Li}_{2}\left(\frac{1}{1-\beta}\right)-2 \operatorname{Li}_{2}(1-\beta)+\operatorname{Li}_{2}\left(\frac{1}{\beta+1}\right)\right. \\
& \left.-\operatorname{Li}_{2}\left(\frac{\beta+1}{1-\beta}\right)+\frac{1}{2} \operatorname{Li}_{2}\left(\frac{4 m_{t}^{2}}{s}\right)-\frac{1}{2} \operatorname{Li}_{2}\left(\frac{s}{4 m_{t}^{2}}\right)+\frac{\pi^{2}}{6}\right)+\frac{\pi^{2} C_{10}}{6 K^{2} s}-\frac{C_{11}}{K s_{1}} \ln \left(-\frac{s}{\mu^{2}}\right) \\
& +\frac{1}{K s_{1}}\left((t-u) \ln \left(\frac{m_{t}^{2}}{\mu^{2}}\right) m_{t}^{2}-s_{1} t_{1} \ln \left(\frac{t_{1}}{\mu^{2}}\right)\right) .
\end{aligned}
$$

The IR-divergent parts are proportional to the tree amplitudes

$$
\begin{aligned}
& \mathcal{A}_{b 1}\left(\lambda_{1}, \lambda_{2}, \lambda_{3}, \lambda_{4}\right)=\mathcal{A}_{\text {tree }}\left(\lambda_{1}, \lambda_{2}, \lambda_{3}, \lambda_{4}\right) \frac{\alpha_{s}}{4 \pi}\left\{-\frac{2}{\epsilon_{\mathrm{IR}}^{2}}+\frac{2}{\epsilon_{\mathrm{IR}}}\left(2 \ln \left(\frac{t_{1}}{\mu^{2}}\right)-\ln \left(\frac{m_{t}^{2}}{\mu^{2}}\right)\right)\right\}+\cdots \\
& \mathcal{A}_{b 2}\left(\lambda_{1}, \lambda_{2}, \lambda_{3}, \lambda_{4}\right)=\mathcal{A}_{\text {tree }}\left(\lambda_{1}, \lambda_{2}, \lambda_{3}, \lambda_{4}\right) \frac{\alpha_{s}}{4 \pi}\left\{\frac{2}{\epsilon_{\mathrm{IR}}^{2}}-\frac{2}{\epsilon_{\mathrm{IR}}}\left(2 \ln \left(\frac{u_{1}}{\mu^{2}}\right)-\ln \left(\frac{m_{t}^{2}}{\mu^{2}}\right)\right)\right\}+\cdots
\end{aligned}
$$

As mentioned before, the SM results presented above agree with those in ref. [32].

\section{KK Gluon Induced Helicity Amplitude for $q \bar{q} \rightarrow t \bar{t}$}

\section{Results for LO Diagrams}

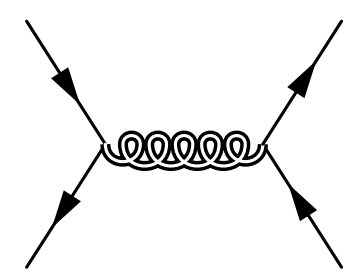

FIG. 6: KK gluon induced tree graph for $q \bar{q} \rightarrow t \bar{t}$. Diagrams vanish identically are not shown.

The fermionic current coupled to the massive color octet is

$$
\bar{\psi} \gamma^{\mu}\left(C_{L}^{I} P_{L}+C_{R}^{I} P_{R}\right) \psi
$$

where $I=q, t$ denotes a massless quark or massive top quark, respectively. And $P_{L, R}=\left(1 \mp \gamma_{5}\right) / 2$ are the chiral projection operators. It is straightforward to calculate tree amplitudes (Fig. 6) for the KK-gluon-mediated process. The color structure is identical 
to that of the gluon induced diagrams, and the Lorentz part is written in terms of spinor products

$$
\begin{aligned}
& \mathcal{A}_{\text {tree }, K K}(+,-,+,+)=\frac{2 i C_{R}^{q} m_{t}}{s-m_{K K}^{2}} \frac{C_{R}^{t}\left\langle\eta_{4} 1\right\rangle\left\langle\eta_{3}|\mathbf{3}| 2\right]+C_{L}^{t}\left\langle\eta_{3} 1\right\rangle\left\langle\eta_{4}|4| 2\right]}{\left\langle 3^{b} \eta_{3}\right\rangle\left\langle\eta_{4} 4^{b}\right\rangle}, \\
& \mathcal{A}_{\text {tree } K K}(-,+,+,+)=\frac{2 i C_{L}^{q} m_{t}}{s-m_{K K}^{2}} \frac{C_{R}^{t}\left\langle\eta_{4} 2\right\rangle\left\langle\eta_{3}|\mathbf{3}| 1\right]+C_{L}^{t}\left\langle\eta_{3} 2\right\rangle\left\langle\eta_{4}|\mathbf{4}| 1\right]}{\left\langle 3^{b} \eta_{3}\right\rangle\left\langle\eta_{4} 4^{b}\right\rangle} .
\end{aligned}
$$

At the NLO, chiral magnetic-like interaction

$$
\bar{\psi} \frac{i \sigma^{\mu \nu} q_{\nu}}{2 m_{t}}\left(C_{L}^{I} P_{L}+C_{R}^{I} P_{R}\right) \psi
$$

will be induced from one-loop triangle diagrams. We also provide tree amplitudes which will be used to construct one-loop amplitudes

$$
\begin{aligned}
& \mathcal{A}_{\text {tree }, K K}^{(m)}(+,-,+,+)=-\frac{i C_{L}^{q}}{m_{t}\left(s-m_{K K}^{2}\right)} \frac{C_{R}^{t} m_{t}^{2}[21]\left\langle\eta_{3} 1\right\rangle\left\langle\eta_{4} 1\right\rangle+C_{L}^{t}\langle 12\rangle\left\langle\eta_{3}|\mathbf{3}| 2\right]\left\langle\eta_{4}|\mathbf{4}| 2\right]}{\left\langle 3^{b} \eta_{3}\right\rangle\left\langle\eta_{4} 4^{b}\right\rangle}, \\
& \mathcal{A}_{\text {tree }, K K}^{(m)}(-,+,+,+)=\frac{i C_{R}^{q}}{m_{t}\left(s-m_{K K}^{2}\right)} \frac{C_{R}^{t} m_{t}^{2}[21]\left\langle\eta_{3} 2\right\rangle\left\langle\eta_{4} 2\right\rangle+C_{L}^{t}\langle 12\rangle\left\langle\eta_{3}|\mathbf{3}| 1\right]\left\langle\eta_{4}|\mathbf{4}| 1\right]}{\left\langle 3^{b} \eta_{3}\right\rangle\left\langle\eta_{4} 4^{b}\right\rangle} .
\end{aligned}
$$

2. Results for Self-energy Diagrams
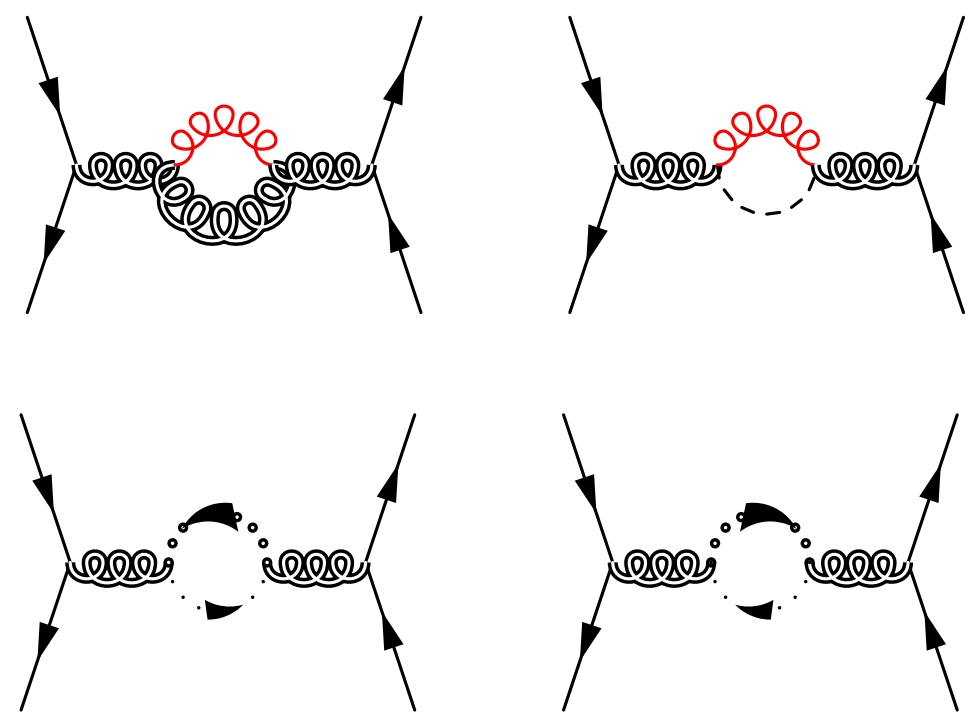

FIG. 7: KK gluon induced one-loop self energy graphs for $q \bar{q} \rightarrow t \bar{t}$. Diagrams vanish identically are not shown.

At the NLO in QCD coupling $\alpha_{s}$, the massive KK gluon propagator (Fig: 7) will receive corrections from loop of gauge bosons, their ghosts and $A_{5}^{(1)}$. There will be two Lorentz 
tensor structures $g^{\mu \nu}$ and $q^{\mu} q^{\nu}$, but the latter does not contribute to the amplitude by means of both vector-current and axial-current conservation on the massless quark side.

The color structure is identical to the tree amplitudes. We have found for the Lorentz part

$$
\begin{aligned}
& \mathcal{A}_{s f, K K}\left(\lambda_{1}, \lambda_{2}, \lambda_{3}, \lambda_{4}\right)=\mathcal{A}_{\text {tree }, K K}\left(\lambda_{1}, \lambda_{2}, \lambda_{3}, \lambda_{4}\right) \frac{\alpha_{s}}{\pi} \frac{s}{s-m_{K K}^{2}}\left\{\frac{1}{\epsilon_{\mathrm{UV}}}\left(\frac{9 m_{K K}^{2}}{4 s}+\frac{5}{2}\right)\right. \\
& +\frac{1}{12 s^{3}}\left[3 \ln \left(\frac{m_{K K}^{2}}{\mu^{2}}\right)\left(2 m_{K K}^{4}-6 s m_{K K}^{2}-15 s^{2}\right) m_{K K}^{2}\right. \\
& \left.\left.-6 \ln \left(\frac{m_{K K}^{2}-s}{\mu^{2}}\right)\left(m_{K K}^{6}-3 s m_{K K}^{4}-3 s^{2} m_{K K}^{2}+5 s^{3}\right)+s\left(-6 m_{K K}^{4}+51 s m_{K K}^{2}+56 s^{2}\right)\right]\right\} .
\end{aligned}
$$

We subtract the one-loop KK gluon propagator on the mass shell, and obtain mass renormalization and wave-function renormalization:

$$
\begin{aligned}
& \delta m_{K K}^{2}=m_{K K}^{2} \frac{\alpha_{s}}{\pi}\left\{-\frac{19}{4 \epsilon_{\mathrm{UV}}}+\frac{19}{4} \ln \left(\frac{m_{K K}^{2}}{\mu^{2}}\right)-\frac{101}{12}\right\} \\
& \delta Z_{K K}=\frac{\alpha_{s}}{\pi}\left\{\frac{5}{2 \epsilon_{\mathrm{UV}}}-\frac{3}{2 \epsilon_{\mathrm{IR}}}-\ln \left(\frac{m_{K K}^{2}}{\mu^{2}}\right)+\frac{13}{6}\right\} .
\end{aligned}
$$

We choose $\overline{\mathrm{MS}}$ scheme to renormalize the coupling between quarks and the massive KK gluon. The counter-term contribution that cancels the UV-divergent part of KK gluon self-energy is given by

$$
\begin{aligned}
\mathcal{A}_{s f, K K}^{C T}\left(\lambda_{1}, \lambda_{2}, \lambda_{3}, \lambda_{4}\right)= & \mathcal{A}_{\text {tree }, K K}\left(\lambda_{1}, \lambda_{2}, \lambda_{3}, \lambda_{4}\right) \frac{\alpha_{s}}{\pi}\left\{-\delta Z_{K K}^{\overline{\mathrm{MS}}}+\frac{\delta m_{K K}^{2}}{s-m_{K K}^{2}}\right\} \\
= & \mathcal{A}_{\text {tree }, K K}\left(\lambda_{1}, \lambda_{2}, \lambda_{3}, \lambda_{4}\right) \frac{\alpha_{s}}{\pi}\left\{-\frac{5}{2 \epsilon_{\mathrm{UV}}}+\frac{5}{2} \ln \left(\frac{m_{K K}^{2}}{\mu^{2}}\right)\right. \\
& \left.+\frac{m_{K K}^{2}}{s-m_{K K}^{2}}\left(-\frac{19}{4 \epsilon_{\mathrm{UV}}}+\frac{19}{4} \ln \left(\frac{m_{K K}^{2}}{\mu^{2}}\right)-\frac{101}{12}\right)\right\} .
\end{aligned}
$$

Here we have include a logarithmic term $\frac{5}{2} \ln \left(\frac{m_{K K}^{2}}{\mu^{2}}\right)$ in the definition of $\delta Z_{K K}^{\overline{\mathrm{MS}}}, \delta Z_{K K}^{\overline{\mathrm{MS}}}=$ $\frac{\alpha_{s}}{\pi}\left(\frac{5}{2 \epsilon_{\mathrm{UV}}}-\frac{5}{2} \ln \frac{m_{K K}^{2}}{\mu^{2}}\right)$.

\section{Results for Triangle Diagrams}

For the one-loop massless triangle diagrams (Fig. 8), the Lorentz amplitude is again the tree amplitude multiplied by a form factor. The form factor is the same for both helicity 

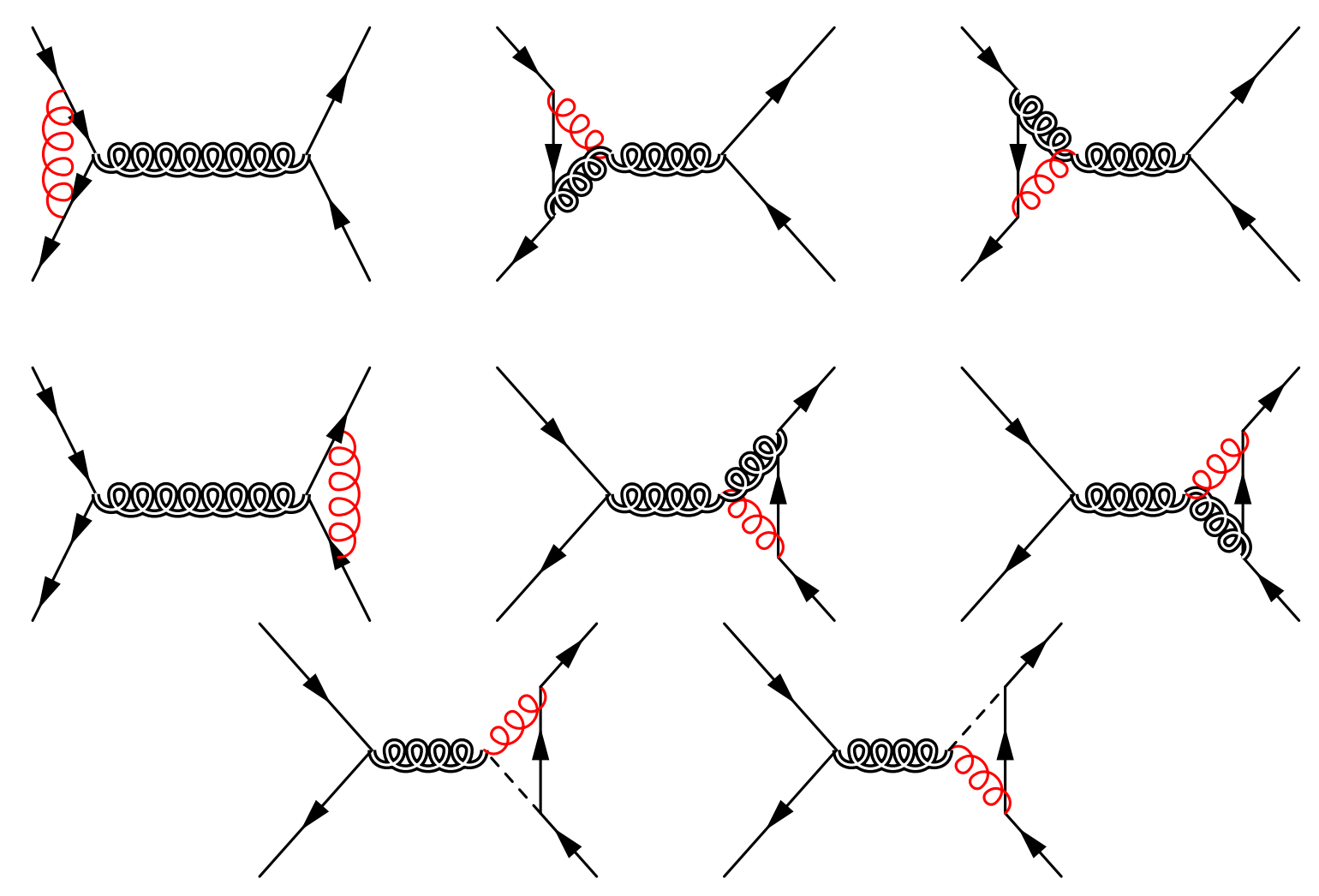

FIG. 8: KK gluon induced one-loop triangle graphs for $q \bar{q} \rightarrow t \bar{t}$. Diagrams vanish identically are not shown.

configurations $(+,-,+,+)$ and $(-,+,+,+)$. The explicit result is

$$
\begin{aligned}
& \mathcal{A}_{v t, K K}^{q}\left(\lambda_{1}, \lambda_{2}, \lambda_{3}, \lambda_{4}\right)=\mathcal{A}_{\text {tree }, K K}\left(\lambda_{1}, \lambda_{2}, \lambda_{3}, \lambda_{4}\right) \frac{\alpha_{s}}{\pi}\left\{\frac{53}{24 \epsilon_{\mathrm{UV}}}+\frac{1}{12 \epsilon_{\mathrm{IR}}^{2}}\right. \\
& +\frac{1}{\epsilon_{\mathrm{IR}}}\left[-\frac{3 m_{K K}^{2}}{2 s} \ln \left(\frac{m_{K K}^{2}-s}{m_{K K}^{2}}\right)-\frac{1}{12} \ln \left(-\frac{s}{\mu^{2}}\right)-\frac{4}{3}\right]+\frac{1}{24} \ln ^{2}\left(-\frac{s}{\mu^{2}}\right)-\frac{1}{8} \ln \left(-\frac{s}{\mu^{2}}\right) \\
& +\frac{3}{2} C_{0}\left(0,0, s, m_{K K}^{2}, 0,0\right) m_{K K}^{2}-\frac{3 m_{K K}^{2}}{4 s}+\ln \left(\frac{m_{K K}^{2}-s}{\mu^{2}}\right)\left(\frac{3}{4}-\frac{3 m_{K K}^{4}}{4 s^{2}}\right) \\
& \left.+\frac{3}{4} \ln \left(\frac{m_{K K}^{2}}{\mu^{2}}\right)\left(\frac{m_{K K}^{4}}{s^{2}}-2\right)+\frac{25}{24}\right\}
\end{aligned}
$$

where the one-loop scalar integrals $C_{0}[38]$ should be understood as only retaining the finite part. The results for one-loop massive quark vertex amplitudes can be expressed as linear 
combination of tree amplitudes, of both helicity configurations. They have the form

$$
\begin{aligned}
\mathcal{A}_{v t, K K}^{t}(+,-,+,+)= & \frac{2 i C_{R}^{q} m_{t}}{s-m_{K K}^{2}} \frac{\alpha_{s}}{\pi} \frac{1}{\left\langle 3^{b} \eta_{3}\right\rangle\left\langle\eta_{4} 4^{b}\right\rangle}\left\{B_{1}^{K K}\left(C_{R}^{t}\left\langle\eta_{4} 1\right\rangle\left\langle\eta_{3}|\mathbf{3}| 2\right]+C_{L}^{t}\left\langle\eta_{3} 1\right\rangle\left\langle\eta_{4}|\mathbf{4}| 2\right]\right)\right. \\
& +B_{2}^{K K}\left(C_{R}^{t}+C_{L}^{t}\right)\left(\left\langle\eta_{4} 1\right\rangle\left\langle\eta_{3}|\mathbf{3}| 2\right]+\left\langle\eta_{3} 1\right\rangle\left\langle\eta_{4}|\mathbf{4}| 2\right]\right) \\
& \left.+B_{3}^{K K}\left(C_{R}^{t}+C_{L}^{t}\right)\left(m_{t}^{2}[21]\left\langle\eta_{3} 1\right\rangle\left\langle\eta_{4} 1\right\rangle+\langle 12\rangle\left\langle\eta_{3}|\mathbf{3}| 2\right]\left\langle\eta_{4}|\mathbf{4}| 2\right]\right)\right\}, \\
\mathcal{A}_{v t, K K}^{t}(-,+,+,+)= & \frac{2 i C_{L}^{q} m_{t}}{s-m_{K K}^{2}} \frac{\alpha_{s}}{\pi} \frac{1}{\left\langle 3^{b} \eta_{3}\right\rangle\left\langle\eta_{4} 4^{b}\right\rangle}\left\{B_{1}^{K K}\left(C_{R}^{t}\left\langle\eta_{4} 2\right\rangle\left\langle\eta_{3}|\mathbf{3}| 1\right]+C_{L}^{t}\left\langle\eta_{3} 2\right\rangle\left\langle\eta_{4}|\mathbf{4}| 1\right]\right)\right. \\
& +B_{2}^{K K}\left(C_{R}^{t}+C_{L}^{t}\right)\left(\left\langle\eta_{4} 2\right\rangle\left\langle\eta_{3}|\mathbf{3}| 1\right]+\left\langle\eta_{3} 2\right\rangle\left\langle\eta_{4}|\mathbf{4}| 1\right]\right) \\
& \left.-B_{3}^{K K}\left(C_{R}^{t}+C_{L}^{t}\right)\left(m_{t}^{2}[21]\left\langle\eta_{3} 2\right\rangle\left\langle\eta_{4} 2\right\rangle+\langle 12\rangle\left\langle\eta_{3}|\mathbf{3}| 1\right]\left\langle\eta_{4}|\mathbf{4}| 1\right]\right)\right\},
\end{aligned}
$$

where $B_{i}^{K K}, i=1,2,3$ are coefficients that depend on $s, m_{t}^{2}$ and $m_{K K}^{2}$. We give explicit expressions for these coefficients. Only $B_{1}^{K K}$ has divergent parts. The other 2 coefficients are finite. The first coefficient is

$$
\begin{aligned}
& B_{1}^{K K}=\frac{53}{24 \epsilon_{\mathrm{UV}}}+\left(\frac{2 m_{t}^{2}}{s}-1\right) \frac{1}{12 \beta \epsilon_{\mathrm{IR}}} \ln \left(\frac{\beta+1}{\beta-1}\right)+\frac{C_{1}^{K K}}{24 s s_{1}}+\frac{s-2 m_{t}^{2}}{12} C_{0}\left(m_{t}^{2}, m_{t}^{2}, s, m_{t}^{2}, 0, m_{t}^{2}\right) \\
& +\frac{3 C_{2}^{K K}}{2 s s_{1}} C_{0}\left(m_{t}^{2}, m_{t}^{2}, s, m_{K K}^{2}, m_{t}^{2}, 0\right)+\frac{C_{3}^{K K}}{6 s s_{1}} \ln \left(\frac{m_{t}^{2}}{\mu^{2}}\right)+\frac{3\left(m_{K K}^{2}+8 m_{t}^{2}+s\right) m_{K K}^{2}}{4 s s_{1}} \ln \left(\frac{m_{K K}^{2}}{\mu^{2}}\right) \\
& +\frac{3\left(m_{K K}^{2}+8 m_{t}^{2}+s\right)\left(s-m_{K K}^{2}\right)}{4 s s_{1}} \ln \left(\frac{m_{K K}^{2}-s}{\mu^{2}}\right)-\frac{3\left(2 m_{K K}^{2} m_{t}^{2}-m_{K K}^{2} s+2 m_{t}^{2} s-2 s^{2}\right)}{4 s s_{1}} \\
& \times B_{0}\left(m_{t}^{2}, m_{K K}^{2}, m_{t}^{2}\right)-\frac{\left(4 m_{t}^{2}-3 s\right)}{24 s_{1}} B_{0}\left(s, m_{t}^{2}, m_{t}^{2}\right),
\end{aligned}
$$

with polynomial coefficients

$$
\begin{aligned}
& C_{1}^{K K}=47 s^{2}-36 m_{K K}^{2} s-420 m_{t}^{2} s+72 m_{K K}^{2} m_{t}^{2}, \\
& C_{2}^{K K}=m_{t}^{2} m_{K K}^{4}+s^{2} m_{K K}^{2}-2 s m_{t}^{2} m_{K K}^{2}-3 s^{2} m_{t}^{2}, \\
& C_{3}^{K K}=-8 s^{2}+7 m_{t}^{2} s-9 m_{K K}^{2} m_{t}^{2} .
\end{aligned}
$$

The second coefficient is

$$
\begin{aligned}
& B_{2}^{K K}=-\frac{C_{4}^{K K}}{12 s s_{1}^{2}}-\frac{m_{t}^{2}}{12 s_{1}} B_{0}\left(s, m_{t}^{2}, m_{t}^{2}\right)+\frac{3 C_{5}^{K K}}{4 s s_{1}^{2}} B_{0}\left(m_{t}^{2}, m_{K K}^{2}, m_{t}^{2}\right)+\frac{3 C_{8}^{K K} m_{K K}^{2}}{4 s s_{1}^{2}} \ln \left(\frac{m_{K K}^{2}}{\mu^{2}}\right) \\
& -\frac{C_{7}^{K K} m_{t}^{2}}{12 s s_{1}^{2}} \ln \left(\frac{m_{t}^{2}}{\mu^{2}}\right)-\frac{3 C_{6}^{K K}\left(s-m_{K K}^{2}\right) m_{t}^{2}}{2 s s_{1}^{2}} C_{0}\left(m_{t}^{2}, m_{t}^{2}, s, m_{K K}^{2}, m_{t}^{2}, 0\right) \\
& +\frac{3\left(s-m_{K K}^{2}\right) m_{t}^{2}\left(3 m_{K K}^{2}+8 m_{t}^{2}-5 s\right)}{2 s s_{1}^{2}} \ln \left(\frac{m_{K K}^{2}-s}{\mu^{2}}\right),
\end{aligned}
$$


with polynomial coefficients

$$
\begin{aligned}
& C_{4}^{K K}=-72 m_{K K}^{2} m_{t}^{4}+224 s m_{t}^{4}-110 s^{2} m_{t}^{2}+108 s m_{K K}^{2} m_{t}^{2}-9 s^{2} m_{K K}^{2}, \\
& C_{5}^{K K}=-4 m_{K K}^{2} m_{t}^{4}-4 s^{2} m_{t}^{2}+4 m_{t}^{4} s+8 s m_{K K}^{2} m_{t}^{2}-s^{2} m_{K K}^{2}, \\
& C_{6}^{K K}=2 m_{t}^{2} s-2 s^{2}+m_{K K}^{2} s+2 m_{K K}^{2} m_{t}^{2}, \\
& C_{7}^{K K}=-53 s^{2}+18 m_{K K}^{2} s+104 m_{t}^{2} s+36 m_{K K}^{2} m_{t}^{2}, \\
& C_{8}^{K K}=16 m_{t}^{4}+6 m_{K K}^{2} m_{t}^{2}-6 s m_{t}^{2}-s^{2} .
\end{aligned}
$$

The third coefficient is

$$
\begin{aligned}
& B_{3}^{K K}=\frac{C_{9}^{K K}}{24 s m_{t}^{2} s_{1}^{2}}+\frac{1}{24 s_{1}} B_{0}\left(s, m_{t}^{2}, m_{t}^{2}\right)+\frac{C_{12}^{K K}}{24 s s_{1}^{2}} \ln \left(\frac{m_{t}^{2}}{\mu^{2}}\right)+\frac{3 C_{11}^{K K}\left(s-m_{K K}^{2}\right)}{4 s s_{1}^{2}} \\
& \times C_{0}\left(m_{t}^{2}, m_{t}^{2}, s, m_{K K}^{2}, m_{t}^{2}, 0\right)-\frac{3\left(s-m_{K K}^{2}\right)\left(-3 m_{K K}^{2}+8 m_{t}^{2}+s\right)}{4 s s_{1}^{2}} \ln \left(\frac{m_{K K}^{2}-s}{\mu^{2}}\right) \\
& -\frac{3 C_{10}^{K K}}{8 s m_{t}^{2} s_{1}^{2}} B_{0}\left(m_{t}^{2}, m_{K K}^{2}, m_{t}^{2}\right)-\frac{3 C_{13}^{K K} m_{K K}^{2}}{8 s m_{t}^{2} s_{1}^{2}} \ln \left(\frac{m_{K K}^{2}}{\mu^{2}}\right),
\end{aligned}
$$

with polynomial coefficients

$$
\begin{aligned}
& C_{9}^{K K}=72 m_{K K}^{2} m_{t}^{4}+80 s m_{t}^{4}+34 s^{2} m_{t}^{2}-108 s m_{K K}^{2} m_{t}^{2}+9 s^{2} m_{K K}^{2}, \\
& C_{10}^{K K}=4 m_{K K}^{2} m_{t}^{4}+12 s m_{t}^{4}-8 s m_{K K}^{2} m_{t}^{2}+s^{2} m_{K K}^{2}, \\
& C_{11}^{K K}=-2 m_{t}^{2} m_{K K}^{2}-s m_{K K}^{2}+6 s m_{t}^{2} \\
& C_{12}^{K K}=19 s^{2}-18 m_{K K}^{2} s+32 m_{t}^{2} s-36 m_{K K}^{2} m_{t}^{2} \\
& C_{13}^{K K}=16 m_{t}^{4}-6 m_{K K}^{2} m_{t}^{2}-2 s m_{t}^{2}+s^{2} .
\end{aligned}
$$

Here the scalar one-loop integrals $B_{0}$ 's and $C_{0}$ 's [38] should be understood as only retaining the finite part.

The renormailzation of the above two one-loop vertex amplitudes are very similar to the case of the SM process. We choose to renormalize the chiral coupling between the KK gluon and quarks in the $\overline{\mathrm{MS}}$ scheme

$$
\delta Z_{C_{L, R}^{q / t}}=-\delta Z_{\Gamma_{K K}}^{\overline{\mathrm{MS}}}-\delta Z_{q / t}^{\overline{\mathrm{MS}}}-\frac{1}{2} \delta Z_{K K}^{\overline{\mathrm{MS}}},
$$

where $\delta Z_{\Gamma_{K K}}^{\overline{\mathrm{MS}}}=\frac{\alpha_{s}}{\pi} \frac{53}{24 \epsilon_{\mathrm{UV}}}$ is the UV-divergent part of the one-loop vertex function, which is common regardless of the quark mass, and also regardless of the chirality of the coupling. And $\delta Z_{q / t}^{\overline{\mathrm{MS}}}$ is just the UV-divergent part of the on-shell wave-function renormalization constant for massless or massive quark. 
The counter-term contributions are easily obtained:

$$
\begin{aligned}
\mathcal{A}_{v t, K K}^{q, C T}\left(\lambda_{1}, \lambda_{2}, \lambda_{3}, \lambda_{4}\right) & =\mathcal{A}_{\text {tree }, K K}\left(\lambda_{1}, \lambda_{2}, \lambda_{3}, \lambda_{4}\right) \times\left(-\delta Z_{\Gamma_{K K}}^{\overline{\mathrm{MS}}}-\delta Z_{q}^{\overline{\mathrm{MS}}}+\delta Z_{q}^{\mathrm{OS}}\right) \\
& =\mathcal{A}_{\text {tree }, K K}\left(\lambda_{1}, \lambda_{2}, \lambda_{3}, \lambda_{4}\right) \frac{\alpha_{s}}{\pi}\left\{-\frac{53}{24 \epsilon_{\mathrm{UV}}}+\frac{1}{3 \epsilon_{\mathrm{IR}}}\right\} \\
\mathcal{A}_{v t, K K}^{t, C T}\left(\lambda_{1}, \lambda_{2}, \lambda_{3}, \lambda_{4}\right) & =\mathcal{A}_{\text {tree }, K K}\left(\lambda_{1}, \lambda_{2}, \lambda_{3}, \lambda_{4}\right) \times\left(-\delta Z_{\Gamma_{K K}}^{\overline{\mathrm{MS}}}-\delta Z_{t}^{\overline{\mathrm{MS}}}+\delta Z_{t}^{\mathrm{OS}}\right) \\
& =\mathcal{A}_{\text {tree }, K K}\left(\lambda_{1}, \lambda_{2}, \lambda_{3}, \lambda_{4}\right) \frac{\alpha_{s}}{\pi}\left\{-\frac{53}{24 \epsilon_{\mathrm{UV}}}-\frac{2}{3 \epsilon_{\mathrm{IR}}}+\ln \left(\frac{m_{t}^{2}}{\mu^{2}}\right)-\frac{5}{3}\right\} .
\end{aligned}
$$

4. Results for Box Diagrams
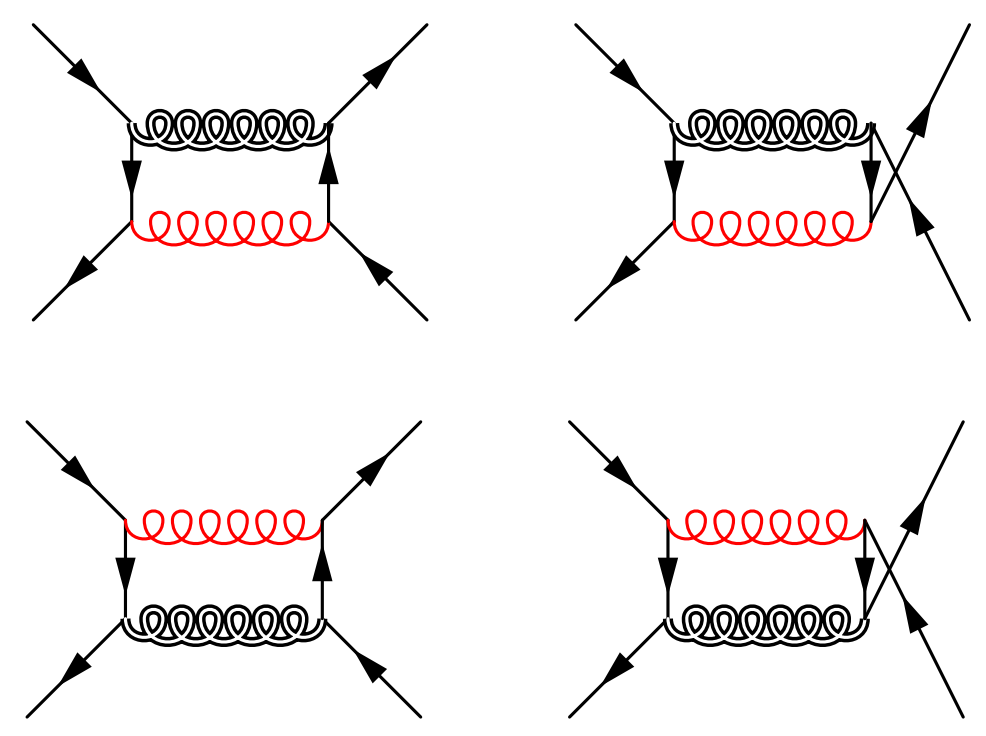

FIG. 9: KK gluon induced regular and cross box diagrams. Diagrams vanish identically are not shown.

For the KK gluon-mediated process at one-loop, there are 2 regular box diagrams and 2 crossed box diagrams (Fig. 9). We do not repeat the color structure which is identical to that of the Standard Model box diagrams. The Lorentz part can be expressed in terms of the same set of spinor products basis combined with both left- and right-handed couplings, with a total of 6 coefficients $B_{i, 1 / 2}^{K K}, i=4,5,6$ which depend on $s, t, u, m_{t}^{2}, m_{K K}^{2}$. For the 
regular box diagrams with both helicity configurations, the amplitudes are written as

$$
\begin{aligned}
\mathcal{A}_{b 1, K K}(+,-,+,+)= & \frac{2 i C_{R}^{q} m_{t}}{\left\langle 3^{b} \eta_{3}\right\rangle\left\langle\eta_{4} 4^{b}\right\rangle} \frac{\alpha_{s}}{\pi}\left\{\left(B_{4,1}^{K K} C_{R}^{t}\left\langle\eta_{4} 1\right\rangle\left\langle\eta_{3}|\mathbf{3}| 2\right]+B_{4,2}^{K K} C_{L}^{t}\left\langle\eta_{3} 1\right\rangle\left\langle\eta_{4}|\mathbf{4}| 2\right]\right)\right. \\
& +\left(B_{5,1}^{K K} C_{L}^{t}\left\langle\eta_{4} 1\right\rangle\left\langle\eta_{3}|\mathbf{3}| 2\right]+B_{5,2}^{K K} C_{R}^{t}\left\langle\eta_{3} 1\right\rangle\left\langle\eta_{4}|\mathbf{4}| 2\right]\right) \\
& \left.+\left(B_{6,1}^{K K} C_{R}^{t}+B_{6,2}^{K K} C_{L}^{t}\right)\left(m_{t}^{2}[21]\left\langle\eta_{3} 1\right\rangle\left\langle\eta_{4} 1\right\rangle+\langle 12\rangle\left\langle\eta_{3}|\mathbf{3}| 2\right]\left\langle\eta_{4}|\mathbf{4}| 2\right]\right)\right\}, \\
\mathcal{A}_{b 1, K K}(-,+,+,+)= & \frac{2 i C_{L}^{q} m_{t}}{\left\langle 3^{b} \eta_{3}\right\rangle\left\langle\eta_{4} 4^{b}\right\rangle} \frac{\alpha_{s}}{\pi}\left\{\left(B_{4,2}^{K K} C_{R}^{t}\left\langle\eta_{4} 2\right\rangle\left\langle\eta_{3}|\mathbf{3}| 1\right]+B_{4,1}^{K K} C_{L}^{t}\left\langle\eta_{3} 2\right\rangle\left\langle\eta_{4}|\mathbf{4}| 1\right]\right)\right. \\
& +\left(B_{5,2}^{K K} C_{L}^{t}\left\langle\eta_{4} 2\right\rangle\left\langle\eta_{3}|\mathbf{3}| 1\right]+B_{5,1}^{K K} C_{R}^{t}\left\langle\eta_{3} 2\right\rangle\left\langle\eta_{4}|\mathbf{4}| 1\right]\right) \\
& \left.-\left(B_{6,2}^{K K} C_{R}^{t}+B_{6,1}^{K K} C_{L}^{t}\right)\left(m_{t}^{2}[21]\left\langle\eta_{3} 2\right\rangle\left\langle\eta_{4} 2\right\rangle+\langle 12\rangle\left\langle\eta_{3}|\mathbf{3}| 1\right]\left\langle\eta_{4}|\mathbf{4}| 1\right]\right)\right\} .
\end{aligned}
$$

For the crossed box diagrams the amplitudes are related. We denote $\tilde{B}_{i, 1 / 2}^{K K}$ by exchanging $t$ and $u$

$$
\tilde{B}_{i, 1 / 2}^{K K}=-B_{i, 1 / 2}^{K K}(t \longleftrightarrow u)
$$

Amplitudes for crossed box diagrams are in a similar form

$$
\begin{aligned}
\mathcal{A}_{b 2, K K}(+,-,+,+)= & \frac{2 i C_{R}^{q} m_{t}}{\left\langle 3^{b} \eta_{3}\right\rangle\left\langle\eta_{4} 4^{b}\right\rangle} \frac{\alpha_{s}}{\pi}\left\{\left(\tilde{B}_{4,2}^{K K} C_{R}^{t}\left\langle\eta_{4} 1\right\rangle\left\langle\eta_{3}|\mathbf{3}| 2\right]+\tilde{B}_{4,1}^{K K} C_{L}^{t}\left\langle\eta_{3} 1\right\rangle\left\langle\eta_{4}|\mathbf{4}| 2\right]\right)\right. \\
& +\left(\tilde{B}_{5,2}^{K K} C_{L}^{t}\left\langle\eta_{4} 1\right\rangle\left\langle\eta_{3}|\mathbf{3}| 2\right]+\tilde{B}_{5,1}^{K K} C_{R}^{t}\left\langle\eta_{3} 1\right\rangle\left\langle\eta_{4}|\mathbf{4}| 2\right]\right) \\
& \left.+\left(\tilde{B}_{6,2}^{K K} C_{R}^{t}+\tilde{B}_{6,1}^{K K} C_{L}^{t}\right)\left(m_{t}^{2}[21]\left\langle\eta_{3} 1\right\rangle\left\langle\eta_{4} 1\right\rangle+\langle 12\rangle\left\langle\eta_{3}|\mathbf{3}| 2\right]\left\langle\eta_{4}|\mathbf{4}| 2\right]\right)\right\}, \\
\mathcal{A}_{b 2, K K}(-,+,+,+)= & \frac{2 i C_{L}^{q} m_{t}}{\left\langle 3^{b} \eta_{3}\right\rangle\left\langle\eta_{4} 4^{b}\right\rangle} \frac{\alpha_{s}}{\pi}\left\{\left(\tilde{B}_{4,1}^{K K} C_{R}^{t}\left\langle\eta_{4} 2\right\rangle\left\langle\eta_{3}|\mathbf{3}| 1\right]+\tilde{B}_{4,2}^{K K} C_{L}^{t}\left\langle\eta_{3} 2\right\rangle\left\langle\eta_{4}|\mathbf{4}| 1\right]\right)\right. \\
& +\left(\tilde{B}_{5,1}^{K K} C_{L}^{t}\left\langle\eta_{4} 2\right\rangle\left\langle\eta_{3}|\mathbf{3}| 1\right]+\tilde{B}_{5,2}^{K K} C_{R}^{t}\left\langle\eta_{3} 2\right\rangle\left\langle\eta_{4}|\mathbf{4}| 1\right]\right) \\
& \left.-\left(\tilde{B}_{6,1}^{K K} C_{R}^{t}+\tilde{B}_{6,2}^{K K} C_{L}^{t}\right)\left(m_{t}^{2}[21]\left\langle\eta_{3} 2\right\rangle\left\langle\eta_{4} 2\right\rangle+\langle 12\rangle\left\langle\eta_{3}|\mathbf{3}| 1\right]\left\langle\eta_{4}|\mathbf{4}| 1\right]\right)\right\} .
\end{aligned}
$$

There is no UV divergence, and IR divergence is proportional to the treel amplitudes

$$
\begin{aligned}
\mathcal{A}_{b 1, K K}\left(\lambda_{1}, \lambda_{2}, \lambda_{3}, \lambda_{4}\right)= & \mathcal{A}_{\text {tree }, K K}\left(\lambda_{1}, \lambda_{2}, \lambda_{3}, \lambda_{4}\right) \frac{\alpha_{s}}{4 \pi}\left\{-\frac{2}{\epsilon_{\mathrm{IR}}^{2}}+\frac{2}{\epsilon_{\mathrm{IR}}}\left(2 \ln \left(\frac{t_{1}}{\mu^{2}}\right)\right.\right. \\
& \left.\left.-\ln \left(\frac{m_{t}^{2}}{\mu^{2}}\right)+\frac{2 m_{K K}^{2}}{s} \ln \left(\frac{m_{K K}^{2}-s}{m_{K K}^{2}}\right)\right)\right\}+\cdots \\
\mathcal{A}_{b 2, K K}\left(\lambda_{1}, \lambda_{2}, \lambda_{3}, \lambda_{4}\right)= & \mathcal{A}_{\text {tree }, K K}\left(\lambda_{1}, \lambda_{2}, \lambda_{3}, \lambda_{4}\right) \frac{\alpha_{s}}{4 \pi}\left\{\frac{2}{\epsilon_{\mathrm{IR}}^{2}}-\frac{2}{\epsilon_{\mathrm{IR}}}\left(2 \ln \left(\frac{u_{1}}{\mu^{2}}\right)\right.\right. \\
& \left.\left.-\ln \left(\frac{m_{t}^{2}}{\mu^{2}}\right)+\frac{2 m_{K K}^{2}}{s} \ln \left(\frac{m_{K K}^{2}-s}{m_{K K}^{2}}\right)\right)\right\}+\cdots
\end{aligned}
$$


Next we give explicit results for coefficients $B_{i, 1 / 2}^{K K}, i=4,5,6$. The first two coefficients have the same IR-divergent part. The rest of them are finite. The first coefficient is

$$
\begin{aligned}
B_{4,1}^{K K}= & \frac{1}{s-m_{K K}^{2}}\left[-\frac{1}{2 \epsilon_{\mathrm{IR}}^{2}}+\frac{1}{\epsilon_{\mathrm{IR}}}\left(\ln \left(\frac{t_{1}}{\mu^{2}}\right)-\frac{1}{2} \ln \left(\frac{m_{t}^{2}}{\mu^{2}}\right)+\frac{m_{K K}^{2}}{s} \ln \left(\frac{m_{K K}^{2}-s}{m_{K K}^{2}}\right)\right)\right] \\
& +\frac{C_{14}^{K K}}{2 K^{2}} C_{0}\left(0,0, s, m_{K K}^{2}, 0,0\right)-\frac{C_{15}^{K K} t_{1}}{2 K^{2}} C_{0}\left(m_{t}^{2}, 0, t, m_{t}^{2}, 0,0\right) \\
& -\frac{C_{15}^{K K} t_{1}}{2 K^{2}} C_{0}\left(m_{t}^{2}, 0, t, m_{t}^{2}, m_{K K}^{2}, 0\right)+\frac{C_{17}^{K} t_{1}}{2 K^{2}} D_{0}\left(m_{t}^{2}, m_{t}^{2}, 0,0, s, t, m_{K K}^{2}, m_{t}^{2}, 0,0\right) \\
& -\frac{t_{1}}{K} \ln \left(\frac{t_{1}}{\mu^{2}}\right)+\frac{C_{16}^{K K}}{2 K^{2} s_{1}} C_{0}\left(m_{t}^{2}, m_{t}^{2}, s, m_{K K}^{2}, m_{t}^{2}, 0\right) \\
& -\frac{C_{18}^{K K}}{K s_{1}} \ln \left(\frac{m_{t}^{2}}{\mu^{2}}\right)-\frac{C_{19}^{K} m_{K K}^{2}}{K s s_{1}} \ln \left(\frac{m_{K K}^{2}}{\mu^{2}}\right)+\frac{C_{19}^{K K}\left(m_{K K}^{2}-s\right)}{K s s_{1}} \ln \left(\frac{m_{K K}^{2}-s}{\mu^{2}}\right) \\
& -\frac{m_{t}^{2}\left(m_{t}^{2}+t\right)}{K s_{1}} B_{0}\left(m_{t}^{2}, m_{K K}^{2}, m_{t}^{2}\right)+\frac{2 m_{t}^{2}\left(m_{t}^{2}+t\right)}{K s_{1}},
\end{aligned}
$$

with the the polynomial coefficients

$$
\begin{aligned}
C_{14}^{K K}= & 4 m_{t}^{8}-9 t m_{t}^{6}-u m_{t}^{6}+10 t^{2} m_{t}^{4}-5 t^{3} m_{t}^{2}+s t m_{K K}^{2} m_{t}^{2}-t^{2} u m_{t}^{2}+t^{4}+t^{2} u^{2}-s t^{2} m_{K K}^{2}, \\
C_{15}^{K K}= & m_{t}^{6}-4 t m_{t}^{4}+3 t^{2} m_{t}^{2}+t m_{K K}^{2} m_{t}^{2}-t^{3}-t^{2} m_{K K}^{2}+t^{2} u, \\
C_{16}^{K K}= & -2 m_{K K}^{2} m_{t}^{8}+6 s m_{t}^{8}+8 t m_{t}^{8}-s^{2} m_{t}^{6}-24 t^{2} m_{t}^{6}+2 t m_{K K}^{2} m_{t}^{6}-12 s t m_{t}^{6}+24 t^{3} m_{t}^{4} \\
& +16 s t^{2} m_{t}^{4}+2 t^{2} m_{K K}^{2} m_{t}^{4}+2 s t m_{K K}^{2} m_{t}^{4}+4 s^{2} t m_{t}^{4}-8 t^{4} m_{t}^{2}-12 s t^{3} m_{t}^{2}-5 s^{2} t^{2} m_{t}^{2} \\
& -2 t^{3} m_{K K}^{2} m_{t}^{2}+2 s t^{2} m_{K K}^{2} m_{t}^{2}-s^{2} t m_{K K}^{2} m_{t}^{2}+2 s t^{4}+2 s^{2} t^{3}+s^{3} t^{2}-s^{2} t^{2} m_{K K}^{2}, \\
C_{17}^{K K}= & 4 m_{t}^{8}-m_{K K}^{2} m_{t}^{6}-9 t m_{t}^{6}-u m_{t}^{6}+10 t^{2} m_{t}^{4}+6 t m_{K K}^{2} m_{t}^{4}-t m_{K K}^{4} m_{t}^{2}-5 t^{3} m_{t}^{2} \\
& -6 t^{2} m_{K K}^{2} m_{t}^{2}-t u m_{K K}^{2} m_{t}^{2}-t^{2} u m_{t}^{2}+t^{4}+t^{2} m_{K K}^{4}+t^{2} u^{2}+2 t^{3} m_{K K}^{2}, \\
C_{18}^{K K}= & m_{t}^{2}\left(m_{t}^{2}+u\right), \\
C_{19}^{K K}= & 2 m_{t}^{4}-t^{2}-t u .
\end{aligned}
$$

The second coefficient is

$$
\begin{aligned}
B_{4,2}^{K K}= & \frac{1}{s-m_{K K}^{2}}\left[-\frac{1}{2 \epsilon_{\mathrm{IR}}^{2}}+\frac{1}{\epsilon_{\mathrm{IR}}}\left(\ln \left(\frac{t_{1}}{\mu^{2}}\right)-\frac{1}{2} \ln \left(\frac{m_{t}^{2}}{\mu^{2}}\right)+\frac{m_{K K}^{2}}{s} \ln \left(\frac{m_{K K}^{2}-s}{m_{K K}^{2}}\right)\right)\right] \\
& +\frac{t_{1} m_{t}^{2}}{2 K} C_{0}\left(m_{t}^{2}, 0, t, m_{t}^{2}, 0,0\right)+\frac{t_{1} m_{t}^{2}}{2 K} C_{0}\left(m_{t}^{2}, 0, t, m_{t}^{2}, m_{K K}^{2}, 0\right) \\
& +\frac{\left(t m_{t}^{2}+u m_{t}^{2}-2 t u\right)}{2 K} C_{0}\left(0,0, s, m_{K K}^{2}, 0,0\right)+\frac{2 m_{t}^{4}+t m_{t}^{2}-u m_{t}^{2}-2 t u}{2 K} \\
& \times C_{0}\left(m_{t}^{2}, m_{t}^{2}, s, m_{K K}^{2}, m_{t}^{2}, 0\right)+\frac{\left(m_{K K}^{2} m_{t}^{2}+t m_{t}^{2}+u m_{t}^{2}-2 t u\right) t_{1}}{2 K} \\
& \times D_{0}\left(m_{t}^{2}, m_{t}^{2}, 0,0, s, t, m_{K K}^{2}, m_{t}^{2}, 0,0\right) .
\end{aligned}
$$


The third coefficient is found to be vanishing. The fourth coefficient is

$$
\begin{aligned}
B_{5,2}^{K K}= & -\frac{\left(m_{K K}^{2}-s\right) m_{t}^{2} C_{20}^{K K}}{2 K^{2} s_{1}} C_{0}\left(m_{t}^{2}, m_{t}^{2}, s, m_{K K}^{2}, m_{t}^{2}, 0\right)+\frac{\left(m_{K K}^{2}-s\right)^{2} m_{t}^{2} t_{1}^{2}}{2 K^{2}} \\
& \times D_{0}\left(m_{t}^{2}, m_{t}^{2}, 0,0, s, t, m_{K K}^{2}, m_{t}^{2}, 0,0\right)+\frac{\left(m_{K K}^{2}-s\right) m_{t}^{2} t_{1}^{2}}{2 K^{2}} C_{0}\left(m_{t}^{2}, 0, t, m_{t}^{2}, 0,0\right) \\
& +\frac{\left(m_{K K}^{2}-s\right) m_{t}^{2} t_{1}^{2}}{2 K^{2}} C_{0}\left(m_{t}^{2}, 0, t, m_{t}^{2}, m_{K K}^{2}, 0\right)+\frac{m_{t}^{2} t_{1}}{K t} \ln \left(\frac{t_{1}}{\mu^{2}}\right)-\frac{s\left(m_{K K}^{2}-s\right) m_{t}^{2} t_{1}}{2 K^{2}} \\
& \times C_{0}\left(0,0, s, m_{K K}^{2}, 0,0\right)+\frac{C_{21}^{K K} m_{t}^{2}}{K t s_{1}} \ln \left(\frac{m_{t}^{2}}{\mu^{2}}\right)-\frac{(t-u) m_{K K}^{2} m_{t}^{2}}{K s s_{1}} \ln \left(\frac{m_{K K}^{2}}{\mu^{2}}\right) \\
& +\frac{(t-u)\left(m_{K K}^{2}-s\right) m_{t}^{2}}{K s s_{1}} \ln \left(\frac{m_{K K}^{2}-s}{\mu^{2}}\right)+\frac{m_{t}^{2}\left(m_{t}^{2}+u\right)}{K s_{1}} B_{0}\left(m_{t}^{2}, m_{K K}^{2}, m_{t}^{2}\right) \\
& -\frac{2 m_{t}^{2}\left(m_{t}^{2}+u\right)}{K s_{1}},
\end{aligned}
$$

with the polynomial coefficients

$$
\begin{aligned}
& C_{20}^{K K}=2 m_{t}^{6}+4 t m_{t}^{4}-2 u m_{t}^{4}-t^{2} m_{t}^{2}-u^{2} m_{t}^{2}-t^{3}+t u^{2}-2 t^{2} u \\
& C_{21}^{K K}=2 m_{t}^{4}+u m_{t}^{2}-t u
\end{aligned}
$$

The fifth coefficient is

$$
\begin{aligned}
B_{6,1}^{K K}= & \frac{C_{22}^{K K}}{4 K^{2}} C_{0}\left(0,0, s, m_{K K}^{2}, 0,0\right)-\frac{t_{1} C_{22}^{K K}}{4 K^{2} s} C_{0}\left(m_{t}^{2}, 0, t, m_{t}^{2}, 0,0\right)-\frac{1}{K} \ln \left(\frac{t_{1}}{\mu^{2}}\right) \\
& +\frac{C_{25}^{K K}\left(m_{K K}^{2}-s\right) t_{1}}{4 K^{2} s} D_{0}\left(m_{t}^{2}, m_{t}^{2}, 0,0, s, t, m_{K K}^{2}, m_{t}^{2}, 0,0\right)+\frac{C_{24}^{K K}}{4 K^{2} s_{1}} \\
& \times C_{0}\left(m_{t}^{2}, m_{t}^{2}, s, m_{K K}^{2}, m_{t}^{2}, 0\right)-\frac{m_{K K}^{2}\left(m_{t}^{2}+t\right)}{K s s_{1}} \ln \left(\frac{m_{K K}^{2}}{\mu^{2}}\right)+\frac{\left(m_{K K}^{2}-s\right)\left(m_{t}^{2}+t\right)}{K s s_{1}} \\
& \times \ln \left(\frac{m_{K K}^{2}-s}{\mu^{2}}\right)-\frac{C_{23}^{K K}}{4 K^{2} s t_{1}} C_{0}\left(m_{t}^{2}, 0, t, m_{t}^{2}, m_{K K}^{2}, 0\right)-\frac{C_{26}^{K K}}{2 K s_{1} t_{1}} \ln \left(\frac{m_{t}^{2}}{\mu^{2}}\right) \\
& -\frac{\left(m_{t}^{4}+2 t m_{t}^{2}+t u\right)}{2 K s_{1} t_{1}} B_{0}\left(m_{t}^{2}, m_{K K}^{2}, m_{t}^{2}\right)+\frac{m_{t}^{4}+2 t m_{t}^{2}+t u}{K s_{1} t_{1}}
\end{aligned}
$$


with the polynomial coefficients

$$
\begin{aligned}
& C_{22}^{K K}= 5 m_{t}^{6}-m_{K K}^{2} m_{t}^{4}-11 t m_{t}^{4}-2 u m_{t}^{4}+8 t^{2} m_{t}^{2}+4 t m_{K K}^{2} m_{t}^{2}+3 t u m_{t}^{2}-2 t^{3}-2 t^{2} m_{K K}^{2} \\
&-t u m_{K K}^{2}-t^{2} u, \\
& C_{23}^{K K}= 3 m_{t}^{10}+m_{K K}^{2} m_{t}^{8}-19 t m_{t}^{8}-2 u m_{t}^{8}+35 t^{2} m_{t}^{6}+6 t m_{K K}^{2} m_{t}^{6}+11 t u m_{t}^{6}-29 t^{3} m_{t}^{4} \\
&-11 t^{2} m_{K K}^{2} m_{t}^{4}-5 t u m_{K K}^{2} m_{t}^{4}-13 t^{2} u m_{t}^{4}+12 t^{4} m_{t}^{2}-2 t^{2} u^{2} m_{t}^{2}+8 t^{3} m_{K K}^{2} m_{t}^{2} \\
&+2 t^{2} u m_{K K}^{2} m_{t}^{2}+5 t^{3} u m_{t}^{2}-2 t^{5}+2 t^{3} u^{2}-2 t^{4} m_{K K}^{2}+2 t^{2} u^{2} m_{K K}^{2}-t^{3} u m_{K K}^{2}-t^{4} u, \\
&= 10 m_{t}^{8}-4 m_{K K}^{2} m_{t}^{6}+7 t m_{t}^{6}-3 u m_{t}^{6}-9 t^{2} m_{t}^{4}-2 u^{2} m_{t}^{4}-3 t m_{K K}^{2} m_{t}^{4}-u m_{K K}^{2} m_{t}^{4} \\
&-5 t u m_{t}^{4}+3 t u^{2} m_{t}^{2}+4 t^{2} m_{K K}^{2} m_{t}^{2}-7 t^{2} u m_{t}^{2}+2 t^{4}+t^{2} u^{2}+2 t^{3} m_{K K}^{2}-t u^{2} m_{K K}^{2} \\
&+3 t^{2} u m_{K K}^{2}+3 t^{3} u, \\
& C_{24}^{K K}=-5 m_{t}^{6}+m_{K K}^{2} m_{t}^{4}+11 t m_{t}^{4}+2 u m_{t}^{4}-8 t^{2} m_{t}^{2}-4 t m_{K K}^{2} m_{t}^{2}-3 t u m_{t}^{2}+2 t^{3}+2 t^{2} m_{K K}^{2} \\
&+t u m_{K K}^{2}+t^{2} u, \\
& C_{25}^{K K}= 3 m_{t}^{4}+2 u m_{t}^{2}-t u . \\
& C_{26}^{K K}=
\end{aligned}
$$

The sixth coefficient is

$$
\begin{aligned}
B_{6,2}^{K K}= & \frac{t_{1}^{2}}{4 K s} C_{0}\left(m_{t}^{2}, 0, t, m_{t}^{2}, 0,0\right)+\frac{\left(m_{K K}^{2}-s\right) t_{1}^{2}}{4 K s} D_{0}\left(m_{t}^{2}, m_{t}^{2}, 0,0, s, t, m_{K K}^{2}, m_{t}^{2}, 0,0\right) \\
& -\frac{t_{1}}{4 K} C_{0}\left(0,0, s, m_{K K}^{2}, 0,0\right)+\frac{m_{t}^{2}+t}{4 K} C_{0}\left(m_{t}^{2}, m_{t}^{2}, s, m_{K K}^{2}, m_{t}^{2}, 0\right) \\
& -\frac{m_{t}^{4}+2 t m_{t}^{2}-t^{2}-2 t u}{4 K s} C_{0}\left(m_{t}^{2}, 0, t, m_{t}^{2}, m_{K K}^{2}, 0\right)
\end{aligned}
$$

Again, all scalar one-loop integrals should be understood as only retaining the finite part.

\section{DISCUSSION AND CONCLUSION}

We have presented the one-loop helicity amplitudes for $t \bar{t}$ production induced by KK gluon. The results are expressed in terms of four independent spinor products. A special feature of our calculation is that only interaction vertices that are uniquely fixed by gauge

symmetry are considered, except the couplings between quark and the first KK mode, which are not fixed by gauge invariance, but are required by the LO process. Choosing the vertices this way allows our calculation to be model independent as much as possible. In fact, except for the couplings between quark and the first KK mode, the only model dependent information are the color representation and the mass of KK gluon. In this way, all the 
infrared QCD effects are captured in our calculation, which usually dominate fixed order cross section [29]. This implies that with minor modification, our calculation results can be applied to a variety of models containing a massive color octet, cf. refs. [25, 39 53]. To confirm this, we also derive the relevant Feynman rules in a model with $S U(3)_{L} \times S U(3)_{R}$ symmetry, which is spontaneously broken to diagonal $S U(3)_{C}$ by a bi-triplet scalar field. As expected, all Feynman rules that are uniquely determined by gauge symmetry are the same in the two models, including those vertices involving ghost and goldstone bosons ${ }^{1}$.

Using the Feynman rules derived in this paper, the decay width of KK gluon can also be calculated to NLO. The relevant Feynman diagrams are depicted in Fig. 10, After including
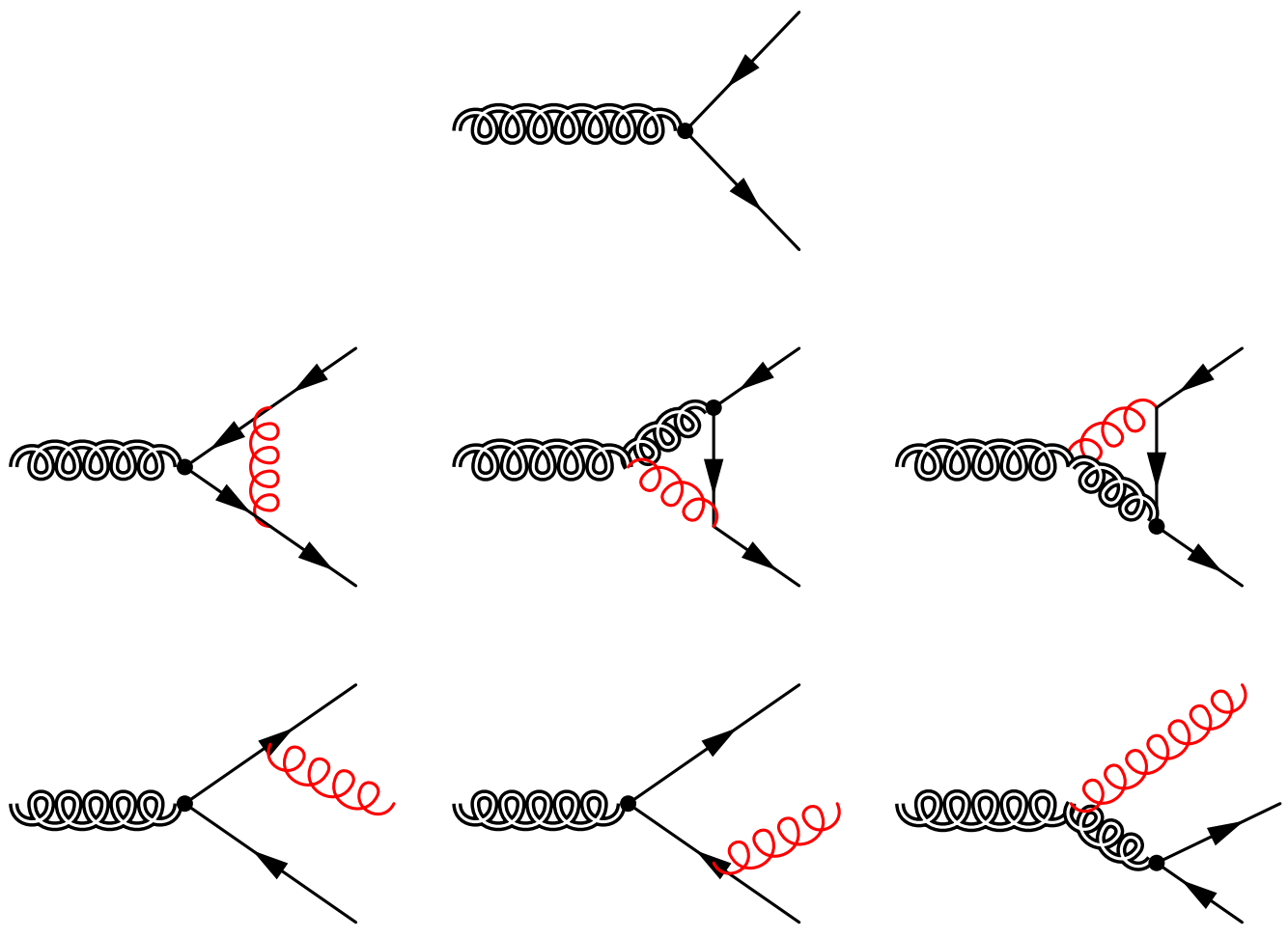

FIG. 10: LO and NLO corrections to total decay width of KK gluon.

both virtual corrections and real emission contributions, the NLO decay width can be written as

$$
\Gamma_{K K}=m_{K K} \sum_{I=u, d, \ldots, t} \frac{\left(C_{L}^{I}\left(\mu_{R}\right)\right)^{2}+\left(C_{R}^{I}\left(\mu_{R}\right)\right)^{2}}{48 \pi}\left[1+\frac{\alpha_{s}}{\pi}\left(\frac{167}{12}-\pi^{2}-\frac{15}{4} \ln \frac{m_{K K}^{2}}{\mu_{R}^{2}}\right)\right],
$$

\footnotetext{
${ }^{1} A_{5}^{(1)}$ plays the role of goldstone boson in RS model.
} 
where $I$ is a quark flavor index, and the corrections of $\mathcal{O}\left(m_{t}^{2} / m_{K K}^{2}\right)$ have been neglected. $C_{L / R}^{I}\left(\mu_{R}\right)$ is the running coupling between quark and KK gluon. The RG-running of $C_{L / R}^{I}(\mu)$ can be read off from Eq. (83) and is given by

$$
\frac{C_{L / R}^{I}(\mu)}{C_{L / R}^{I}\left(\mu_{0}\right)}=\left(\frac{\alpha_{s}(\mu)}{\alpha_{s}\left(\mu_{0}\right)}\right)^{15 /\left(2 \beta_{0}\right)}
$$

where $\beta_{0}=23 / 3$ is the QCD beta function for $N_{C}=3, n_{f}=5$. For large $C_{L / R}^{I}$, the total decay width is large, $\sim 10 \%$. This invalidates the narrow width approximation, and is one of the motivation of this work. A simple framework for dealing with virtual particles with large width is the so-called complex mass scheme [54]. In this scheme, $m_{K K}^{2}$ is complex,

$$
m_{K K}^{2}=\tilde{m}_{K K}^{2}-i \tilde{m}_{K K} \Gamma_{K K}
$$

where $\tilde{m}_{K K}$ is a real mass. All the mass terms in the Feynman rules and in the helicity amplitudes should be understood as complex number.

In conclusion, we have calculated the one-loop amplitudes for $t \bar{t}$ production induced by KK gluon. As mentioned above, the calculation presented in this paper shows for the first time how to calculate renormalized one-loop amplitudes predicted by new physics model. To obtain phenomenological relevant numerical result, we need to combine virtual and real corrections to cancel the remaining IR divergences and obtain a finite cross section. This will be presented elsewhere [20].

\section{Acknowledgments}

We would like to thank Ding Yu Shao for helpful correspondence, and Jing Shu for useful discussion. This work was supported in part by the National Natural Science Foundation of China, under Grants No.11021092 and No.10975004. C.P.Y acknowledges the support of the U.S. National Science Foundation under Grand No. PHY-0855561.

\section{Appendix A: Relevant Feynman Rules}

We collect the relevant Feynman rules that enter our calculation in this appendix. All the momenta are flowing into the vertices in this section. The coupling between the first 
KK mode and SM quark are given by

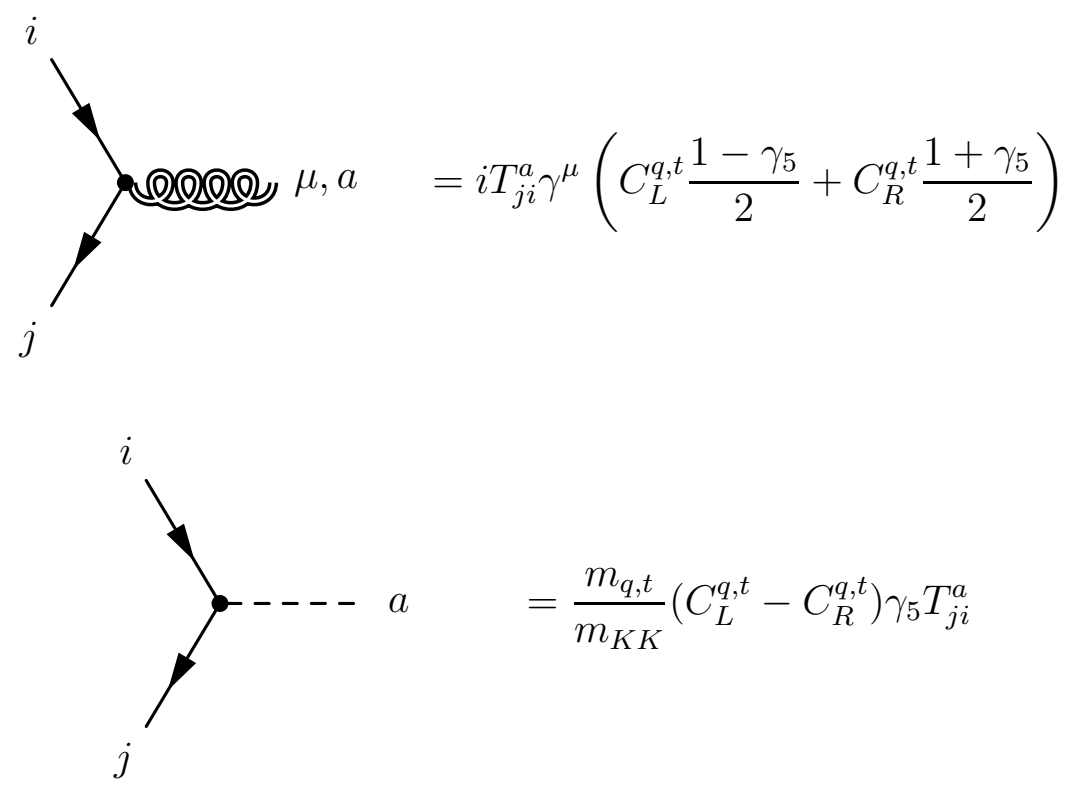

The trilinear and quartic coupling between gluon and KK gluon are given by
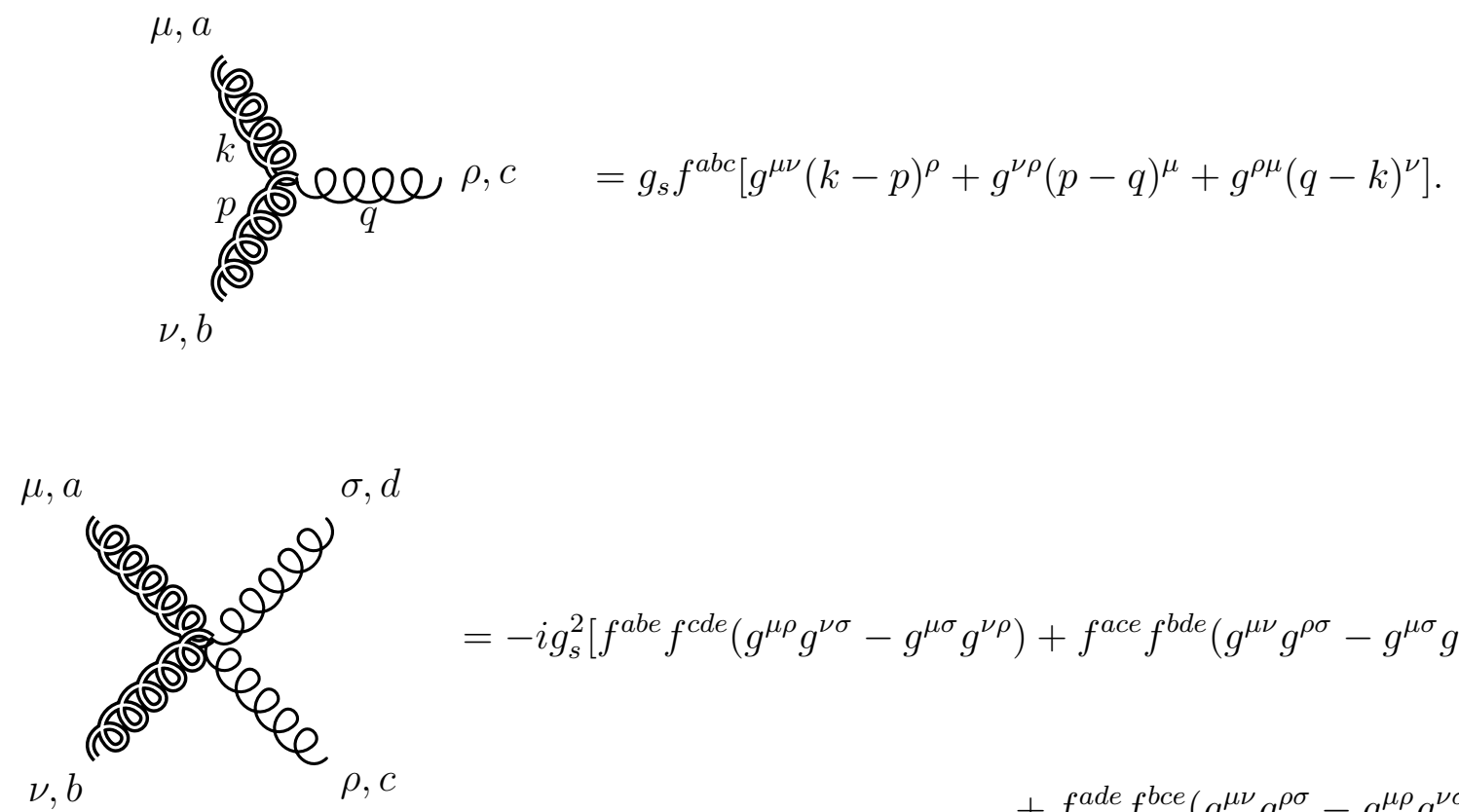

$$
\begin{array}{r}
=-i g_{s}^{2}\left[f^{a b e} f^{c d e}\left(g^{\mu \rho} g^{\nu \sigma}-g^{\mu \sigma} g^{\nu \rho}\right)\right. \\
+f^{a c e} f^{b d e}\left(g^{\mu \nu} g^{\rho \sigma}-g^{\mu \sigma} g^{\nu \rho}\right) \\
\left.+f^{a d e} f^{b c e}\left(g^{\mu \nu} g^{\rho \sigma}-g^{\mu \rho} g^{\nu \sigma}\right)\right]
\end{array}
$$

Coupling between $A_{5}$ and gluon:

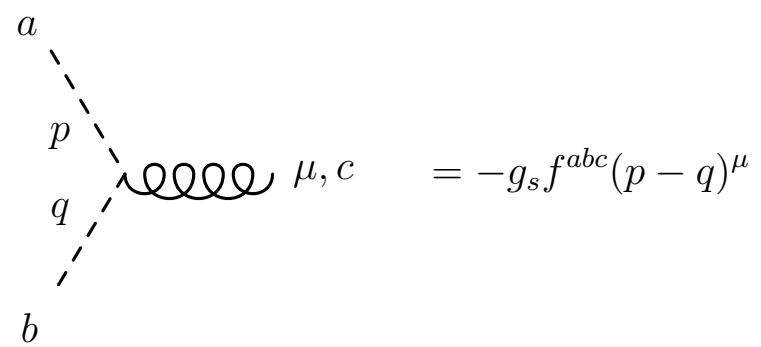




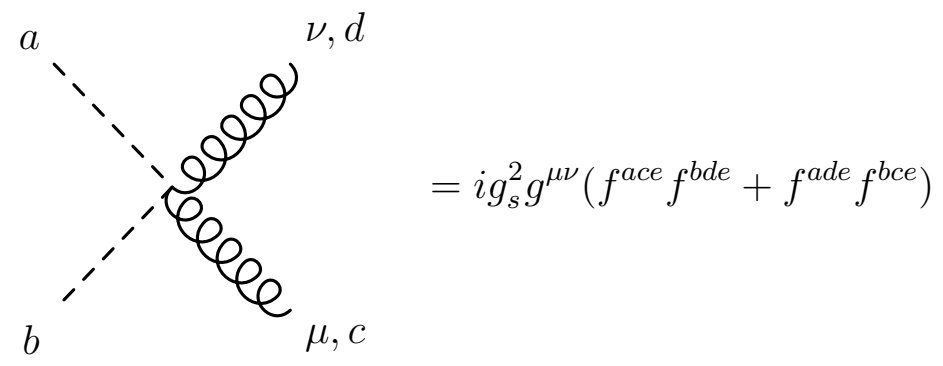

The coupling between gluon, KK gluon and $A_{5}$ :

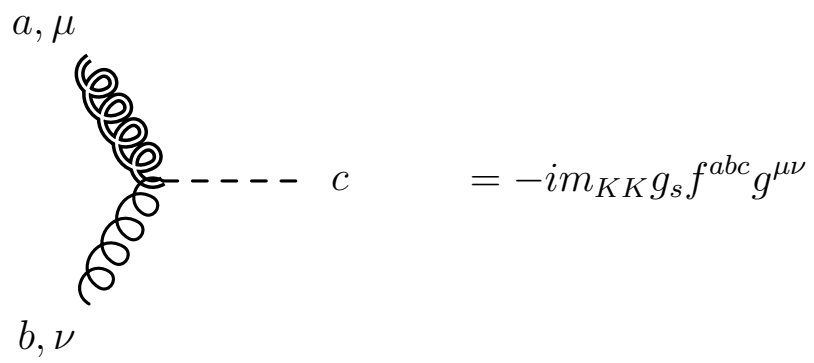

Coupling between ghost of KK gluon and gluon, where the ghost of gluon is denoted as dotted line, and the ghost of KK gluon is denoted as circle line:

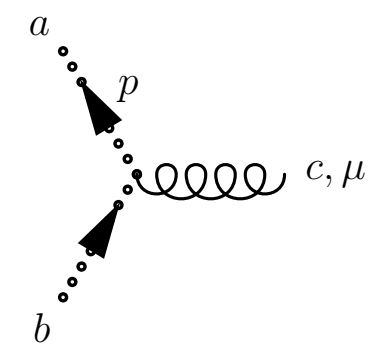

Coupling between ghost of KK gluon, ghost of gluon, and KK gluon:

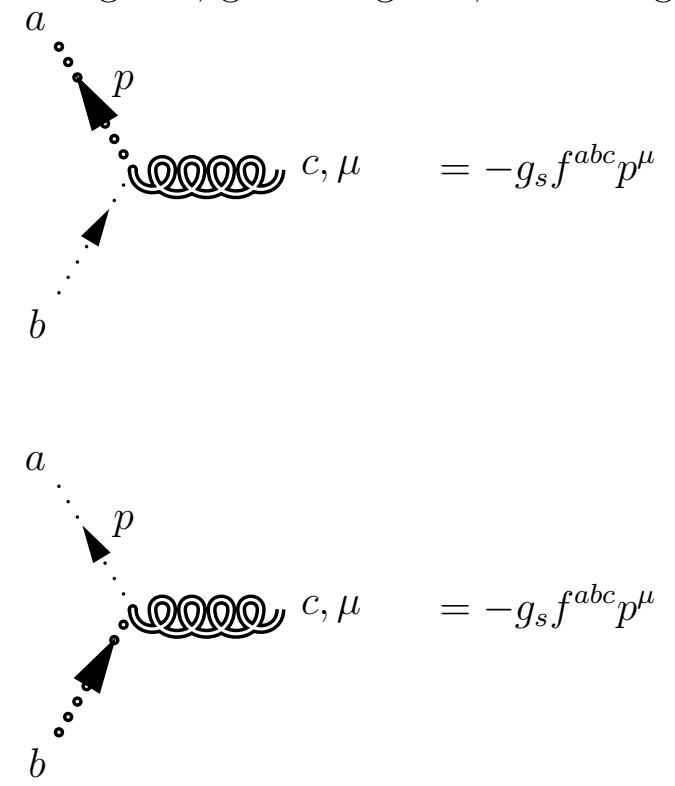




\section{Appendix B: Benchmark numbers for the virtual corrections}

To facilitate a convenient comparison to our calculation, we provide the explicit numbers of squared LO and NLO virtual amplitudes in this appendix for a single phase space point:

$$
\begin{aligned}
& p_{1}=(736.270321435165,0,0,736.270321435165) \\
& p_{2}=(736.270321435165,0,0,-736.270321435165) \\
& p_{3}=(736.270321435165,0,630.456435312587,-338.913586920306) \\
& p_{4}=(736.270321435165,0,-630.456435312587,338.913586920306)
\end{aligned}
$$

where the momentum are given in the unit of $\mathrm{GeV}$. The pole mass of top quark is chosen as 172.5 GeV. The renormalization scale is set to be $\mu=172.5 \mathrm{GeV}$. Strong coupling constant is chosen as $\alpha_{s}(\mu)=0.107663194383306$, corresponds to $\alpha_{s}\left(M_{Z}\right)=0.118$. The complex

mass of KK gluon is given by $m_{K K}^{2}=2250000-362039.269141765 i\left(\mathrm{GeV}^{2}\right)$. Finally the coupling between the quark and KK gluon at the scale $m_{t}$ are

$$
\begin{aligned}
& C_{L}^{q}\left(m_{t}\right)=-1.74, \quad C_{R}^{q}\left(m_{t}\right)=1.74, \\
& C_{L}^{t}\left(m_{t}\right)=1.74, \quad C_{R}^{t}\left(m_{t}\right)=-1.74 .
\end{aligned}
$$

We defined the LO squared amplitude as

$$
\begin{gathered}
\sum_{\text {spin,color }}\left|\mathcal{M}_{\mathrm{SM}}^{(0)}\right|^{2}=a, \\
\sum_{\text {spin,color }}\left|\mathcal{M}_{\mathrm{KK}}^{(0)}\right|^{2}=b,
\end{gathered}
$$

where $\mathcal{M}_{\mathrm{SM}}^{(0)}$ and $\mathcal{M}_{\mathrm{KK}}^{(0)}$ are the LO amplitude for $q \bar{q} \rightarrow t \bar{t}$ induced by gluon and KK gluon, respectively. Although the SM amplitudes are well known, we nonetheless give them here for the convenience of the reader. At the specific phase space point we have chosen, the numbers on the amplitudes are given in Table. [1.

Next we define the virtual squared amplitudes as

$$
\begin{aligned}
\sum_{\text {spin,color }} 2 \operatorname{Re}\left(\mathcal{M}_{\mathrm{SM}}^{(0)} \mathcal{M}_{\mathrm{SM}}^{(1) *}\right) & =\frac{(4 \pi)^{\epsilon}}{\Gamma(1-\epsilon)}\left(\frac{c_{-2}}{\epsilon_{\mathrm{IR}}^{2}}+\frac{c_{-1}}{\epsilon_{\mathrm{IR}}}+c_{0}\right), \\
\sum_{\text {spin,color }} 2 \operatorname{Re}\left(\mathcal{M}_{\mathrm{KK}}^{(0)} \mathcal{M}_{\mathrm{KK}}^{(1) *}\right) & =\frac{(4 \pi)^{\epsilon}}{\Gamma(1-\epsilon)}\left(\frac{d_{-2}}{\epsilon_{\mathrm{IR}}^{2}}+\frac{d_{-1}}{\epsilon_{\mathrm{IR}}}+d_{0}\right),
\end{aligned}
$$


where $\mathcal{M}_{\mathrm{SM}}^{(1)}$ is the SM one-loop virtual corrections, and $\mathcal{M}_{\mathrm{KK}}^{(1)}$ is the one-loop virtual corrections induced by KK gluon, including those diagrams in figures 3, 7, 8, and 9. All the scalar functions with complex argument are evaluated by the Fortran package ONELOOP [55]. The divergent coefficients, $c_{-2}, c_{-1}, d_{-2}$ and $d_{-1}$ are given explicitly in a simple form in the text, so we will not present their numerical values here. The finite terms, $c_{0}$ and $d_{0}$ are given in Table. II.

\begin{tabular}{|l|l|}
\hline$a\left(\mathrm{GeV}^{-2}\right)$ & 18.55003354597 \\
\hline$b\left(\mathrm{GeV}^{-2}\right)$ & 2896.300721184 \\
\hline
\end{tabular}

TABLE I: Numbers on the LO squared amplitude at a specific phase space point.

\begin{tabular}{|c|c|}
\hline$c_{0}\left(\mathrm{GeV}^{-2}\right)$ & -8.084513116805 \\
\hline$d_{0}\left(\mathrm{GeV}^{-2}\right)$ & -1966.611002687 \\
\hline
\end{tabular}

TABLE II: Numbers on the NLO squared amplitude at a specific phase space point.

[1] L. Randall and R. Sundrum, Phys.Rev.Lett. 83, 3370 (1999), hep-ph/9905221.

[2] B. Lillie, L. Randall, and L.-T. Wang, JHEP 09, 074 (2007), hep-ph/0701166.

[3] K. Agashe, A. Belyaev, T. Krupovnickas, G. Perez, and J. Virzi, Phys. Rev. D77, 015003 (2008), hep-ph/0612015.

[4] M. Guchait, F. Mahmoudi, and K. Sridhar, JHEP 05, 103 (2007), hep-ph/0703060.

[5] B. Lillie, J. Shu, and T. M. P. Tait, Phys. Rev. D76, 115016 (2007), 0706.3960.

[6] A. Djouadi, G. Moreau, and R. K. Singh, Nucl. Phys. B797, 1 (2008), 0706.4191.

[7] R. Frederix and F. Maltoni, JHEP 01, 047 (2009), 0712.2355.

[8] U. Baur and L. H. Orr, Phys. Rev. D77, 114001 (2008), 0803.1160.

[9] T. Aaltonen et al. (CDF Collaboration), Phys.Rev.D (2011), 1101.0034.

[10] M. Bowen, S. Ellis, and D. Rainwater, Phys.Rev. D73, 014008 (2006), hep-ph/0509267.

[11] O. Antunano, J. H. Kuhn, and G. Rodrigo, Phys.Rev. D77, 014003 (2008), 0709.1652.

[12] D. Krohn, T. Liu, J. Shelton, and L.-T. Wang (2011), 1105.3743.

[13] A. Djouadi, G. Moreau, and F. Richard (2011), 1105.3158. 
[14] P. Nason, S. Dawson, and R. Ellis, Nucl.Phys. B303, 607 (1988).

[15] W. Beenakker, H. Kuijf, W. van Neerven, and J. Smith, Phys.Rev. D40, 54 (1989).

[16] W. Beenakker, W. van Neerven, R. Meng, G. Schuler, and J. Smith, Nucl.Phys. B351, 507 (1991).

[17] Q.-H. Cao, D. McKeen, J. L. Rosner, G. Shaughnessy, and C. E. Wagner, Phys.Rev. D81, 114004 (2010), 1003.3461.

[18] M. Bauer, F. Goertz, U. Haisch, T. Pfoh, and S. Westhoff, JHEP 1011, 039 (2010), 1008.0742.

[19] B. C. Allanach, F. Mahmoudi, J. P. Skittrall, and K. Sridhar, JHEP 03, 014 (2010), 0910.1350.

[20] H. X. Zhu, C. S. Li, L. Dai, J. Gao, J. Wang, and C.-P. Yuan, work in preparation (2011).

[21] S. Chang, J. Hisano, H. Nakano, N. Okada, and M. Yamaguchi, Phys.Rev. D62, 084025 (2000), hep-ph/9912498.

[22] S. J. Huber and Q. Shafi, Phys.Rev. D63, 045010 (2001), hep-ph/0005286.

[23] T. Gherghetta and A. Pomarol, Nucl.Phys. B586, 141 (2000), hep-ph/0003129.

[24] A. Pomarol, Phys.Lett. B486, 153 (2000), hep-ph/9911294.

[25] H. Davoudiasl, J. Hewett, and T. Rizzo, Phys.Lett. B473, 43 (2000), hep-ph/9911262.

[26] L. Randall and M. D. Schwartz, JHEP 0111, 003 (2001), hep-th/0108114.

[27] H. Novales-Sanchez and J. J. Toscano, Phys. Rev. D82, 116012 (2010), 1008.4638.

[28] A. Flores-Tlalpa, J. Montano, H. Novales-Sanchez, F. Ramirez-Zavaleta, and J. J. Toscano, Phys. Rev. D83, 016011 (2011), 1009.0063.

[29] V. Ahrens, A. Ferroglia, B. D. Pecjak, M. Neubert, and L. L. Yang (2011), 1105.5824.

[30] M. Bohm and W. Hollik, Nucl.Phys. B204, 45 (1982).

[31] M. Czakon and A. Mitov, Nucl.Phys. B824, 111 (2010), 0811.4119.

[32] J. Korner and Z. Merebashvili, Phys.Rev. D66, 054023 (2002), hep-ph/0207054.

[33] S. Badger, R. Sattler, and V. Yundin, Phys.Rev. D83, 074020 (2011), 1101.5947.

[34] Z. Bern, A. De Freitas, L. J. Dixon, and H. Wong, Phys.Rev. D66, 085002 (2002), hep$\mathrm{ph} / 0202271$.

[35] Z. Kunszt, A. Signer, and Z. Trocsanyi, Nucl.Phys. B411, 397 (1994), hep-ph/9305239.

[36] R. Kleiss and W. Stirling, Nucl.Phys. B262, 235 (1985).

[37] S. Badger, J. M. Campbell, and R. Ellis, JHEP 1103, 027 (2011), 1011.6647.

[38] R. K. Ellis and G. Zanderighi, JHEP 02, 002 (2008), 0712.1851.

[39] J. C. Pati and A. Salam, Phys.Rev.Lett. 34, 613 (1975). 
[40] L. J. Hall and A. E. Nelson, Phys.Lett. B153, 430 (1985).

[41] J. L. Hewett and T. G. Rizzo, Phys.Rept. 183, 193 (1989).

[42] C. T. Hill, Phys.Lett. B266, 419 (1991).

[43] K. D. Lane and M. Ramana, Phys.Rev. D44, 2678 (1991).

[44] T. Appelquist, H.-C. Cheng, and B. A. Dobrescu, Phys.Rev. D64, 035002 (2001), hep$\mathrm{ph} / 0012100$.

[45] P. Ferrario and G. Rodrigo, Phys.Rev. D80, 051701 (2009), 0906.5541.

[46] D. E. Morrissey, T. Plehn, and T. M. Tait (2009), 0912.3259.

[47] R. Chivukula, E. H. Simmons, and C.-P. Yuan, Phys.Rev. D82, 094009 (2010), 1007.0260.

[48] B. Xiao, Y.-k. Wang, and S.-h. Zhu (2010), 1011.0152.

[49] Y. Bai, J. L. Hewett, J. Kaplan, and T. G. Rizzo, JHEP 03, 003 (2011), 1101.5203.

[50] A. R. Zerwekh (2011), 1103.0956.

[51] J. Shu, K. Wang, and G. Zhu (2011), 1104.0083.

[52] R. Barcelo, A. Carmona, M. Masip, and J. Santiago (2011), 1105.3333.

[53] U. Haisch and S. Westhoff (2011), 1106.0529.

[54] A. Denner and S. Dittmaier, Nucl. Phys. Proc. Suppl. 160, 22 (2006), hep-ph/0605312.

[55] A. van Hameren (2010), 1007.4716. 